\title{
COAL/POLYMER COPROCESSING WITH EFFICIENT USE OF HYDROGEN
}

Semi-Annual Technical Report

Reporting Period: 03/01/1997 through 08/31/1997

Author: Dr. Linda J. Broadbelt

Report Issue Date: 08/31/1997

DE-FG22-96PC96204

Northwestern University

2145 Sheridan Road

Department of Chemical Engineering

Evanston, IL 60208 


\title{
Disclaimer
}

This report was prepared as an account of work sponsored by an agency of the United States Government. Neither the United States Government nor any agency thereof, nor any of their employees, makes any warranty, express or implied, or assumes any legal liability or responsibility for the accuracy, completeness, or usefulness of any information, apparatus, product, or process disclosed, or represents that its use would not infringe privately owned rights. Reference herein to any specific commercial product, process, or service by trade name, trademark, manufacturer, or otherwise does not necessarily constitute or imply its endorsement, recommendation, or favoring by the United States Government or any agency thereof. The views and opinions of authors expressed herein do not necessarily state or reflect those of the United States Government or any agency thereof.

\begin{abstract}
Recent investigations have demonstrated the feasibility of coprocessing of coal with polymers, with particular interest in employing the polymers as an alternate hydrogen source for coal upgrading and simultaneously recovering high valued fuels and chemicals from plastic waste. A chemical modeling approach was employed in this study in which real and model feedstocks were used to identify the underlying reaction pathways, kinetics, and mechanisms controlling coal liquefaction in the presence of plastics and catalysts. When reacted in binary mixtures, the conversion of tetradecane, a model compound of polyethylene, increased while the selectivities to primary products of 4-(naphthylmethyl) bibenzyl (NBBM) were enhanced. These observations were attributed to the stabilization of NBBM-derived radicals through hydrogen abstraction from tetradecane which in turn, increased the rate of tetradecane conversion.
\end{abstract}

Experiments in the last six months in which the relative concentrations of the components were varied revealed that the effect was indeed a chemical one and not simply a result of dilution. The initial loading of each reactant and overall loading were found to affect both the conversion of tetradecane and the overall product distribution during coprocessing. As the NBBM to tetradecane ratio was raised, only a slight difference in reactant conversions was observed, but a significant change in the selectivity towards primary products from NBBM occurred. The higher proportion of NBBM resulted in an increase of self-interactions, which resulted in a larger quantity of retrograde condensation products. Increasing the initial charge of both reactants resulted in a significant enhancement of tetradecane reactivity. The primary product yields from the coal model compound showed a slight decrease when compared to the reactions conducted with the same reactant ratio but lower total loading. However, the values were higher than those observed for the reactions carried out with a larger ratio of NBBM to tetradecane. These effects on conversions and selectivities were attributed to an increase in bimolecular reactions at higher concentrations.

An experimental protocol was developed to conduct experiments at elevated pressures more representative of coal liquefaction conditions. Preliminary experiments with real feedstocks allowed the extrinsic factors (i.e., diffusion limitations, solvent effects) to be identified. The combination of these two sets of experiments will ultimately be used to carry out process optimization and formulate strategies for catalyst development. 


\section{Table Of Contents}

I. Executive Summary ..................................................

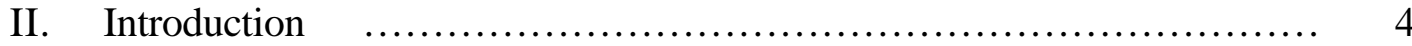

III. Experimental

A. Reactions employing model compounds............................. 5

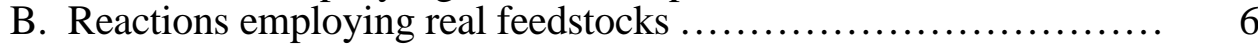

IV. Results and Discussion

A. Model compound studies.......................................... 9

A. 1. Low pressure reactions.................................... 9

A. 1. A. Neat tetradecane pyrolysis ........................... 9

A. 1. B. Neat NBBM pyrolysis............................ 11

A. 1. C. Binary mixture pyrolysis........................... 14

A. 2. High pressure reactions..................................... 19

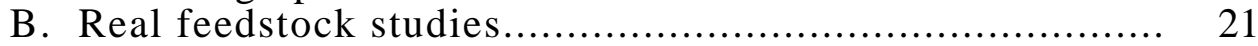





VII. Acknowledgments ............................................... 27

VIII. Manuscripts and Publications............................................... 27



\section{List of Tables}

Table 1. Reaction conditions for low pressure pyrolysis experiments .

\section{List of Figures}

Figure 1. Structure of coal model compound, 4-(naphthylmethyl)bibenzyl .... 5

Figure 2. (A) Flow diagram of pressurizing system. (B) Schematic of gas sampling apparatus

Figure 3. Comparison of conversions of tetradecane and NBBM, neat and binary mixtures, at $420^{\circ} \mathrm{C}$

Figure 4. Comparison of neat tetradecane conversions as a function of reactant loading

Figure 5. Comparison of selectivities of 1-undecene as a function of conversion and reactant loading 
Figure 6. Comparison of selectivities of n-heptane as a function of conversion and reactant loading ..................................................... 12

Figure 7. Proposed mechanism for pyrolysis of tetradecane ................ 13

Figure 8. Bond assignment and corresponding products of NBBM pyrolysis, allowing for only bond fission, hydrogen abstraction, and $\beta$-scission............

Figure 9. Comparison of selectivities of products associated with direct cleavage of bond $\mathrm{A}$ as a function of conversion...

Figure 10. Comparison of products from free radical ipso-substitution reaction which accounts for cleavage of bond A...

Figure 11. Comparison of tetradecane conversions as a function of reactant loading during binary reactions

Figure 12. Comparison of toluene selectivity as a function of reactant loading... $\quad 18$

Figure 13. Comparison of 1-methyl-4-(naphthylmethyl) benzene as a function of reactant loading

Figure 14. Comparison of conversion of NBBM as a function of reactant loading

Figure 15. Comparison of selectivities of phenyl methyl naphthalene as a function of reactant loading and conversion

Figure 16. Comparison of normalized yields of selected $\alpha$-olefins for $20 \mathrm{mg}$ reactant loading of $\mathrm{HDPE}$

Figure 17. Comparison of normalized yields of $\alpha$-olefins as a function of time and carbon number for $20 \mathrm{mg}$ reactant loading of HDPE

Figure 18. Comparison of normalized yields of selected n-alkanes for $20 \mathrm{mg}$ reactant loading of HDPE

Figure 19. Comparison of normalized yields of $n$-alkanes as a function of time and carbon number for $20 \mathrm{mg}$ reactant loading of HDPE

Figure 20. Comparison of normalized yields of tetracosene as a function of reactant loading for reactions with HDPE

Figure 21. Comparison of $\alpha$-olefin to n-alkane ratio for $\mathrm{C}_{18}$ as a function of reactant loading for reactions with $\mathrm{HDPE}$ 


\section{Executive Summary}

The objective of the current research is to investigate the feasibility of coprocessing coal with waste polymers, with particular interest in employing the polymers as an alternate hydrogen source for coal upgrading and simultaneously recovering high valued fuels and chemicals from plastic waste. A chemical modeling approach was employed in which real and model feedstocks were used to identify the underlying reaction pathways, kinetics, and mechanisms controlling coal liquefaction in the presence of plastics and catalysts. Simple model systems were employed to facilitate product analysis and obtain information about the intrinsic reactivity. When reacted in binary mixtures, the conversion of tetradecane, a model compound of polyethylene, increased while the selectivities to primary products of 4-(naphthylmethyl) bibenzyl were enhanced. Experiments in the last six months in which the relative concentrations of the components were varied revealed that the effect was indeed a chemical one and not simply a result of dilution. An experimental protocol was developed to conduct experiments at elevated pressures more representative of coal liquefaction conditions. Preliminary experiments with real feedstocks allowed the extrinsic factors (i.e., diffusion limitations, solvent effects) to be identified. The combination of these two sets of experiments will ultimately be used to carry out process optimization and formulate strategies for catalyst development.

\section{Introduction}

The exploration of new strategies for viable plastics resource recovery is driven by concerns over the inadequacy of current treatment and disposal methods for mixed plastic wastes. The recent emphasis has shifted from sheer disposal to obtaining high-valued, useful products. Post-consumer waste plastics have been targeted since they are a major contributor to the municipal solid waste (MSW) stream, constituting approximately $11 \%$ by weight and $21 \%$ by volume of waste in landfills. ${ }^{1}$ Landfilling is becoming a less viable option since over $40 \%$ of the landfills in the United States were closed in the past decade, and it is estimated that over half of the remaining ones will be full by the end of the century. ${ }^{2}$ With no immediate decrease in the usage of plastic products in sight, this poses a significant dilemma.

Although industry has become increasingly involved, the current motivation for the recovery of plastics is primarily due to government mandates. Efforts have been initiated at all levels of government. Some states, such as California, Oregon, and Wisconsin, have passed laws which specify that plastic bottles must be manufactured from a minimum of $25 \%$ recycled plastics. Germany has dictated that over $80 \%$ of all plastic packaging must be recycled by methods other than combustion by $1996 .{ }^{3-5}$ The government action does not address, however, the technical difficulties encountered with conventional plastics recycling technologies, which include costly separation to removal of impurities and contaminants. A consequence of these problems is that products manufactured from recycled polymers are of lower quality and higher cost (approximately $10 \%$ higher for high-density polyethylene (HDPE)) than those from the corresponding virgin polymer. ${ }^{4}$ As a result, in the United States, only about $4 \%$ of 30 million tons of total plastics produced each year is recycled. ${ }^{6}$

Coprocessing of polymeric waste with other materials may provide potential solutions to the deficiencies of current resource recovery processes, including unfavorable process economics. By incorporating polymeric waste as a minor feed into an existing process, variations in plastic supply and composition could be mediated and as a result, allow for continuous operation. One option for coprocessing is to react polymeric waste 
with coal under direct liquefaction conditions. ${ }^{2,7,8}$ Coprocessing of polymeric waste with coal provides for simultaneous conversion of both feedstocks into high-valued fuels and chemicals.

Catalyst-directed liquefaction of plastic and coal mixtures can be employed in order to reduce the process hydrogen requirements and convert the feed macrostructures into useful fuels and chemicals. The underlying motivation for catalyst-directed coliquefaction of polymers and coal is to use the polymers as the hydrogen source and exploit their inherently higher $\mathrm{H} / \mathrm{C}$ ratio. However, this goal requires information about the underlying reaction pathways, kinetics, and mechanisms controlling coal liquefaction in the presence of catalysts and polymeric materials. A series of experiments using model compounds and real feedstocks for both polymeric waste and coal provides the starting point for unraveling the complex, underlying chemistry. Neat pyrolysis and reactions in the presence of hydrogen, in the presence of a hydrogen donor solvent, in the presence of a catalyst, and in a combined atmosphere span the full range of relevant processing environments. Neat and binary mixture pyrolysis reactions both at low and high pressures are the logical starting point in that they provide valuable thermal baseline information from which subsequent experiments may be judged. Results from thermal degradation studies which have been conducted will be discussed in the following sections.

\section{Experimental}

\section{A. Reactions Employing Model Compounds}

In order to obtain information about underlying reaction pathways, kinetics, and mechanisms without the complicating effects of the macrostructure, experiments were performed using model compounds for both coal and polyethylene, a voluminous component of mixed plastic waste. To mimic the structure of coal, 4-(naphthylmethyl) bibenzyl (NBBM) $(\mathrm{MW}=322)$ was used. NBBM contains both condensed and isolated aromatic species connected by short alkyl chains. An added feature is that it contains five different aromatic-aliphatic or aliphatic-aliphatic carbon-carbon bonds. Successful predictions of the relevant primary products for real systems using NBBM confirmed the adequacy of this model compound, and thus, it will be employed in this study. ${ }^{9-12}$ The structure of NBBM is depicted in Figure 1.

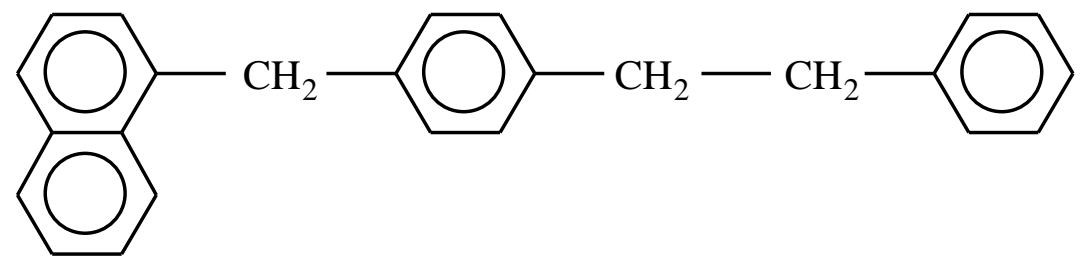

Figure 1: Structure of coal model compound 4-(naphthylmethyl) bibenzyl.

Although numerous hydrocarbons may serve as appropriate model compounds for high density and low density polyethylene, tetradecane, $\mathrm{C}_{14} \mathrm{H}_{30}(\mathrm{MW}=198)$, was chosen as an appropriate compromise in reactant size.

The model compound experiments have been conducted at both low and high pressure to span the range of relevant processing conditions. Low pressure batch pyrolyses of these model compounds have been conducted in $2 \mathrm{ml}$ glass cryules (Wheaton). In addition, preliminary high pressure batch pyrolysis of tetradecane has been carried out. High pressure reactions were conducted in $12 \mathrm{ml}$ batch reactors constructed 
from 316 stainless steel fitted with a high temperature/high pressure two-way ball valve in order to trap product gases for analysis by gas chromatography.

For both low and high pressure experiments, reactions were carried out in an isothermal $\left( \pm 1{ }^{\circ} \mathrm{C}\right)$ fluidized sand bath. For the low pressure pyrolyses, the cryules were charged with the appropriate amount of reactant, purged with argon, and sealed using an oxygen/propane flame. Pyrolyses were conducted at both 420 and $500^{\circ} \mathrm{C}$, and reaction times ranged from 5-150 minutes. Specific reaction conditions are summarized in Table 1. Upon completion of the reaction period, the ampoules were removed from the sand bath and permitted to cool at room temperature. Each reaction time was at minimum duplicated and in some cases, three replicates were performed. The reproducibility for each set of experiments is also listed in Table 1.

The yield of gaseous products was obtained by the weight difference of the reactor before and after opening. The liquid and solid reaction products were extracted from the ampoules using $5 \mathrm{ml}$ of methylene chloride, and an external standard (biphenyl) was added. Product identification and quantification, which enabled reactant conversions and product selectivities to be determined, were achieved using an HP 6890 GC/MS and HP 6890 GC equipped with a flame ionization detector, respectively, each employing a Hewlett Packard $30 \mathrm{~m}$ crosslinked 5\% Ph Me Silicone capillary column.

For the high pressure experiments, the reactor was filled with ca. $120 \mathrm{mg}$ of tetradecane $\left(5.05 \times 10^{-2} \mathrm{M}\right)$ and pressurized to $1000 \mathrm{psig}$ (cold) with nitrogen. In order to completely exclude air and oxygen, the reactors were purged with 1000 psig of nitrogen five times prior to reaction. Thermal degradation was conducted at $420^{\circ} \mathrm{C}$ for $20-150$ minutes. Upon completion of the reaction time, the reactor was immersed in ice water to quench the reaction. Gaseous products were collected using a sampling system, and quantified using an HP 5890 GC equipped with a thermal conductivity detector employing a $6 \mathrm{ft}$ stainless steel Porapak Q column (Supelco). Liquid and solid products were extracted with $15 \mathrm{ml}$ of methylene chloride, and analyzed by the same method as the low pressure experiments. A schematic of the pressurizing and coupling system constructed for this project is depicted in Figure 2.

\section{B. Reactions Employing Real Feedstocks}

In addition to studies using model compounds, initial low pressure studies employing high density polyethylene (HDPE) have been conducted. The HDPE was obtained from Aldrich Chemical $\left(\mathrm{M}_{\mathrm{w}} \approx 125,000\right)$ in pellet form. It was necessary to melt and cut the HDPE pellets in order to obtain samples of appropriate weight. Three sets of experiments, using loadings of $12.3,20.0$, and $27.8 \mathrm{mg}$ respectively, were performed at $420^{\circ} \mathrm{C}$ for $30-180$ minutes. At the end of the reaction period, the ampoules were cooled in a room temperature fluidized sand bath.

Overall gaseous product yields were obtained by the same method used for the low pressure model compound studies. The liquid and solid products in the ampoules were washed and extracted with $4 \mathrm{ml}$ of cyclohexane for 2 hours. Quantitative analysis through a series of extractions using different solvent volumes for different lengths of time revealed that this protocol was sufficient to completely extract products of carbon numbers less than 24 and partially extract up to carbon number 35. A subsequent wash with methylene chloride was then conducted for 3 hours. The percent conversion, X, was determined using the equation: 
Table 1: Reaction Conditions for Low Pressure Pyrolysis Experiments

\begin{tabular}{|c|c|c|c|c|c|}
\hline $\begin{array}{c}\text { Reaction } \\
\text { Set }\end{array}$ & $\begin{array}{c}\text { C14 Loading } \\
(\mathrm{mg})\end{array}$ & $\begin{array}{c}\text { NBBM } \\
\text { Loading (mg) }\end{array}$ & $\begin{array}{c}\text { Reaction } \\
\text { Temperature }\left({ }^{\circ} \mathrm{C}\right)\end{array}$ & $\begin{array}{c}\text { Reaction } \\
\text { Times (min) }\end{array}$ & $\begin{array}{c}\text { Reproducibility } \\
(\%)\end{array}$ \\
\hline $\mathrm{C}_{14}-6.2(\mathrm{a})$ & 6.2 & 0.0 & 420 & $20-150$ & \pm 2 \\
\hline $\mathrm{C}_{14}-12.3(\mathrm{a})$ & 12.3 & 0.0 & 420 & $20-150$ & \pm 2 \\
\hline $\mathrm{C}_{14}-20$ & 20.0 & 0.0 & 500 & $5-60$ & \pm 1 \\
\hline $\mathrm{C}_{14}-20$ (a) & 20.0 & 0.0 & 420 & $20-150$ & \pm 1 \\
\hline $\mathrm{C}_{14}-27.8(\mathrm{a})$ & 27.8 & 0.0 & 420 & $20-150$ & \pm 2 \\
\hline NBBM-20 & 0.0 & 20.0 & 500 & $5-60$ & \pm 2 \\
\hline NBBM-20(a) & 0.0 & 20.0 & 420 & $5-150$ & \pm 2 \\
\hline $\operatorname{Bin}(1)$ & 6.2 & 10.0 & 500 & $5-60$ & \pm 1 \\
\hline $\operatorname{Bin}(1 x-1 x)$ & 6.2 & 10.0 & 420 & $5-150$ & \pm 1 \\
\hline $\operatorname{Bin}(1 x-2 x)$ & 6.2 & 20.0 & 420 & $5-150$ & \pm 2 \\
\hline $\operatorname{Bin}(2 x-2 x)$ & 12.3 & 20.0 & 420 & $5-150$ & \pm 2 \\
\hline
\end{tabular}


A

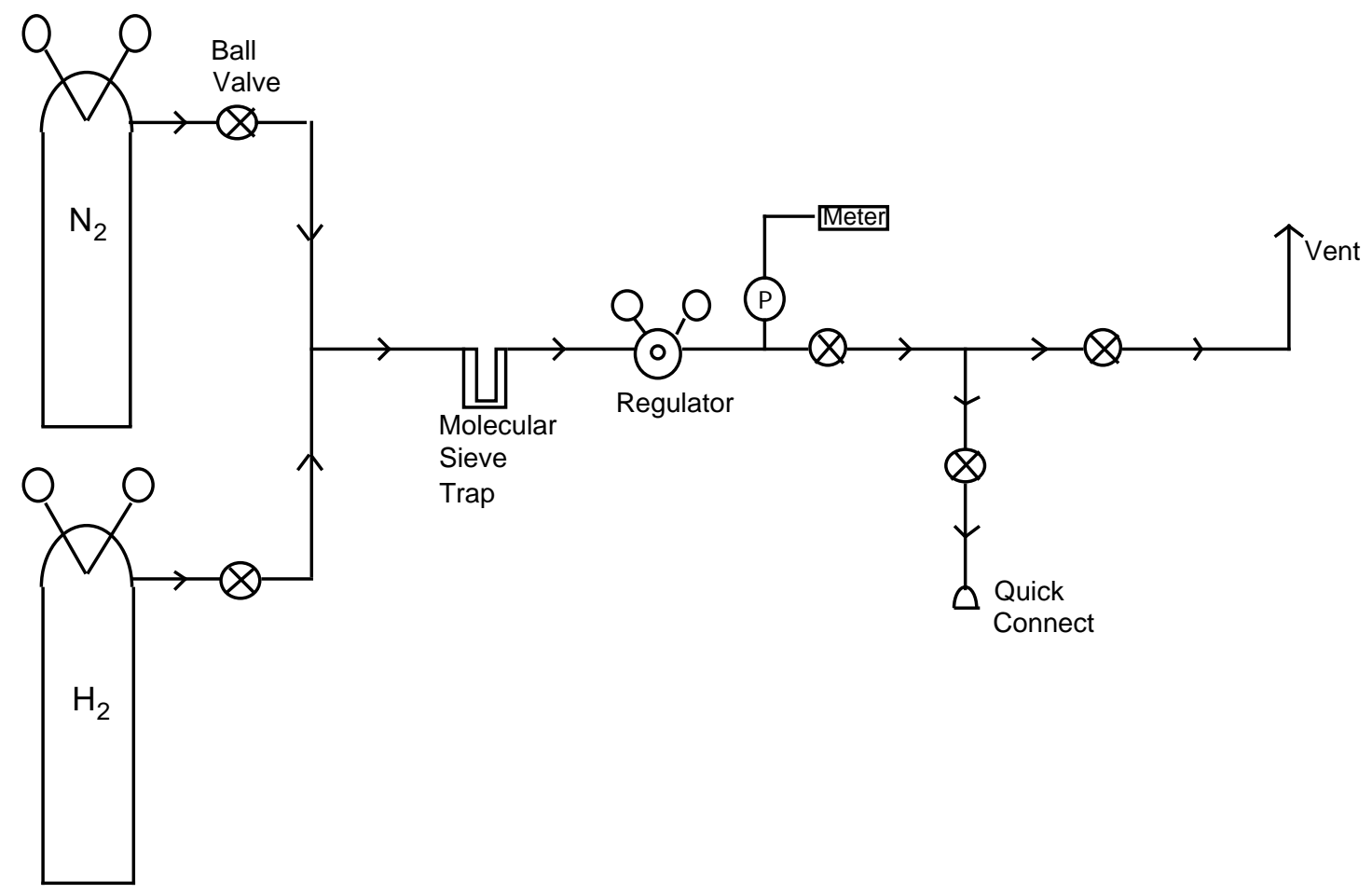

B

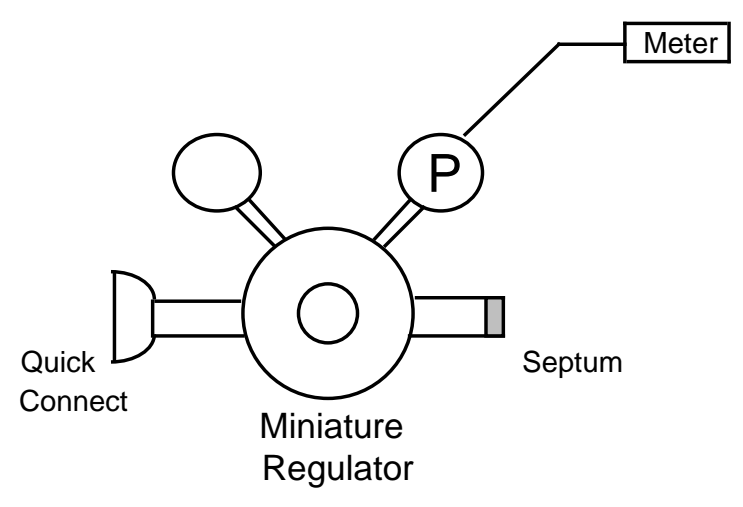

Figure 2: (A) Flow diagram of pressurizing system.

(B) Schematic of gas sampling apparatus. 


$$
\mathrm{X}=\frac{\left(\mathrm{W}_{\mathrm{i}}-\mathrm{MI}\right)}{\mathrm{W}_{\mathrm{i}}} \quad \mathrm{x} \quad 100
$$

where $\mathrm{W}_{\mathrm{i}}$ is the initial weight loading of HDPE and MI is the weight of methylene chloride insoluble material. Products which were soluble in cyclohexane were identified and quantified using the same methods as used during the low pressure model compound experiments.

\section{Results and Discussion}

\section{A. Model Compound Studies}

The model compound experiments at low pressures have been the primary focus of the first year of the project as summarized in Table 1. Quantitative analysis of the reaction products and conversion provided information about the fundamental, underlying chemistry, and mechanistic ideas were put forth to account for the observed behavior. Initial studies at high pressure have thus far been more qualitative, providing information concerning product species and distributions. Results and discussion of both high and low pressure experiments follow in the next sections.

\section{A. 1. Low Pressure Reactions}

Initially, neat and binary mixture pyrolysis experiments of NBBM and tetradecane were conducted at $500{ }^{\circ} \mathrm{C}$. For neat pyrolysis, $20 \mathrm{mg}$ of reactant was used, while for the binary mixtures, equimolar ratios of NBBM to tetradecane based on $10 \mathrm{mg}$ of NBBM were employed. At this temperature, the reactant conversions were very high, as almost complete conversion was achieved at a reaction time of 10 minutes for all the systems studied. As evidenced by the product spectra and the temporal variations of the major products, secondary and tertiary reactions occurred, making it difficult to deconvolute reaction pathways. Therefore, the reaction temperature was lowered to $420^{\circ} \mathrm{C}$, a value still within the range of relevant liquefaction conditions, to achieve lower conversions and isolate primary decomposition pathways. It was noted from the data at $500^{\circ} \mathrm{C}$, however, that the conversion of tetradecane was enhanced slightly in the presence of NBBM as compared to the neat reaction.

Reactions at the loadings above were carried out at $420^{\circ} \mathrm{C}$ and facilitated identification of the underlying reaction pathways and kinetics. As illustrated in Figure 3, the conversion was significantly lower at a given reaction time as compared to that observed at $500^{\circ} \mathrm{C}$. For example, for the same reactant loadings, only $37 \%$ of the tetradecane was converted after 150 minutes of reaction time. Therefore, subsequent reactions were conducted at $420^{\circ} \mathrm{C}$.

\section{A. 1. A. Neat Tetradecane Pyrolysis}

Neat pyrolysis of tetradecane was conducted at loadings ranging from 6.2 to 27.8 $\mathrm{mg}\left(1.56 \times 10^{-2}\right.$ to $\left.7.02 \times 10^{-2} \mathrm{M}\right)$. A plot of conversion versus time for the different concentrations is provided in Figure 4. Overall, the tetradecane conversion increased as the reactant loading was increased. This rate enhancement may be attributed to the increase in the rate of bimolecular reactions at higher reactant loadings, which increases the observed rate of thermal cracking of tetradecane. For the reaction conditions studied, the dependence of the degradation rate on concentration was most pronounced at low loadings, as the rate became relatively insensitive to reactant loading at higher concentrations. 


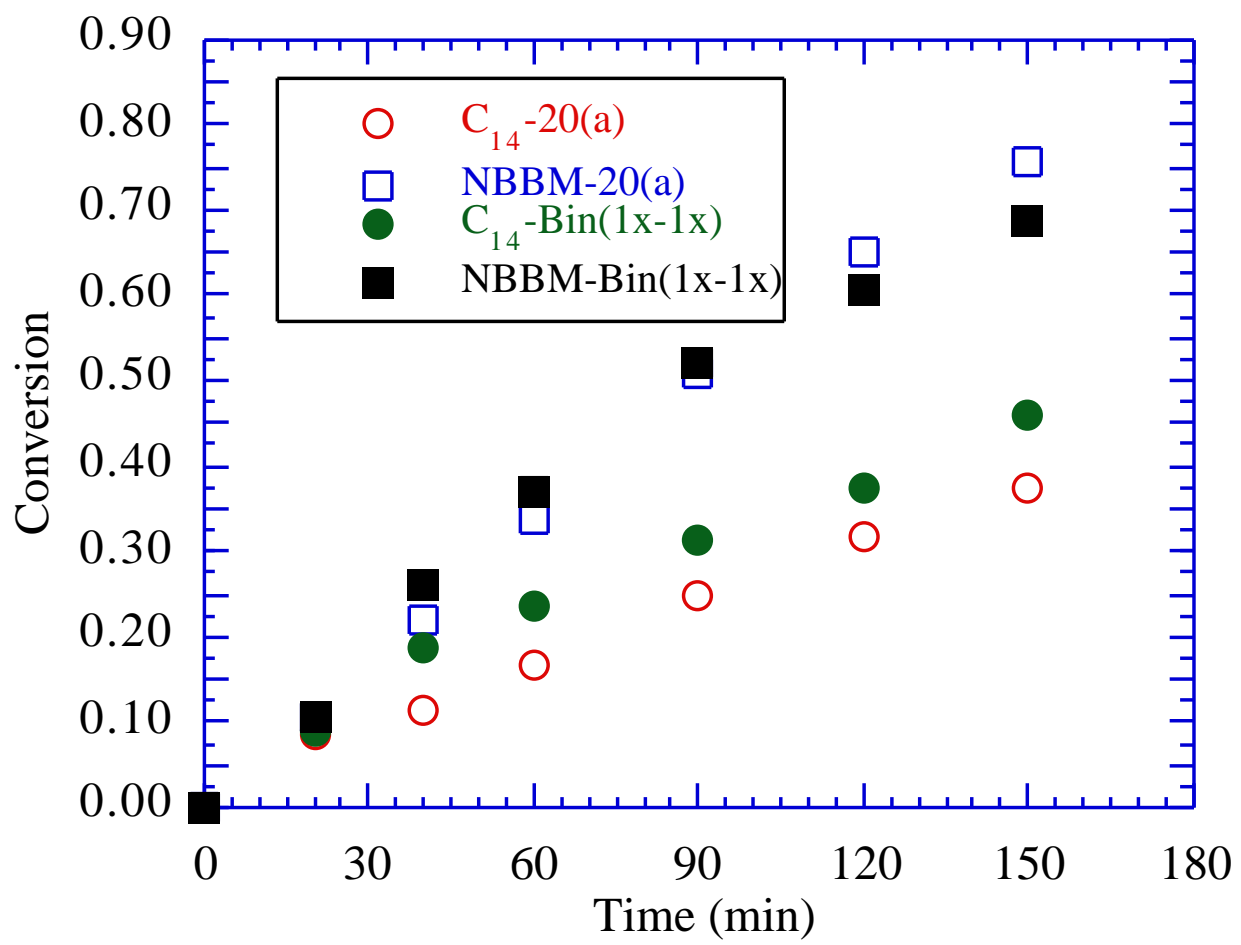

Figure 3: Comparison of conversions of tetradecane and NBBM, neat and in binary mixtures, at $420^{\circ} \mathrm{C}$.

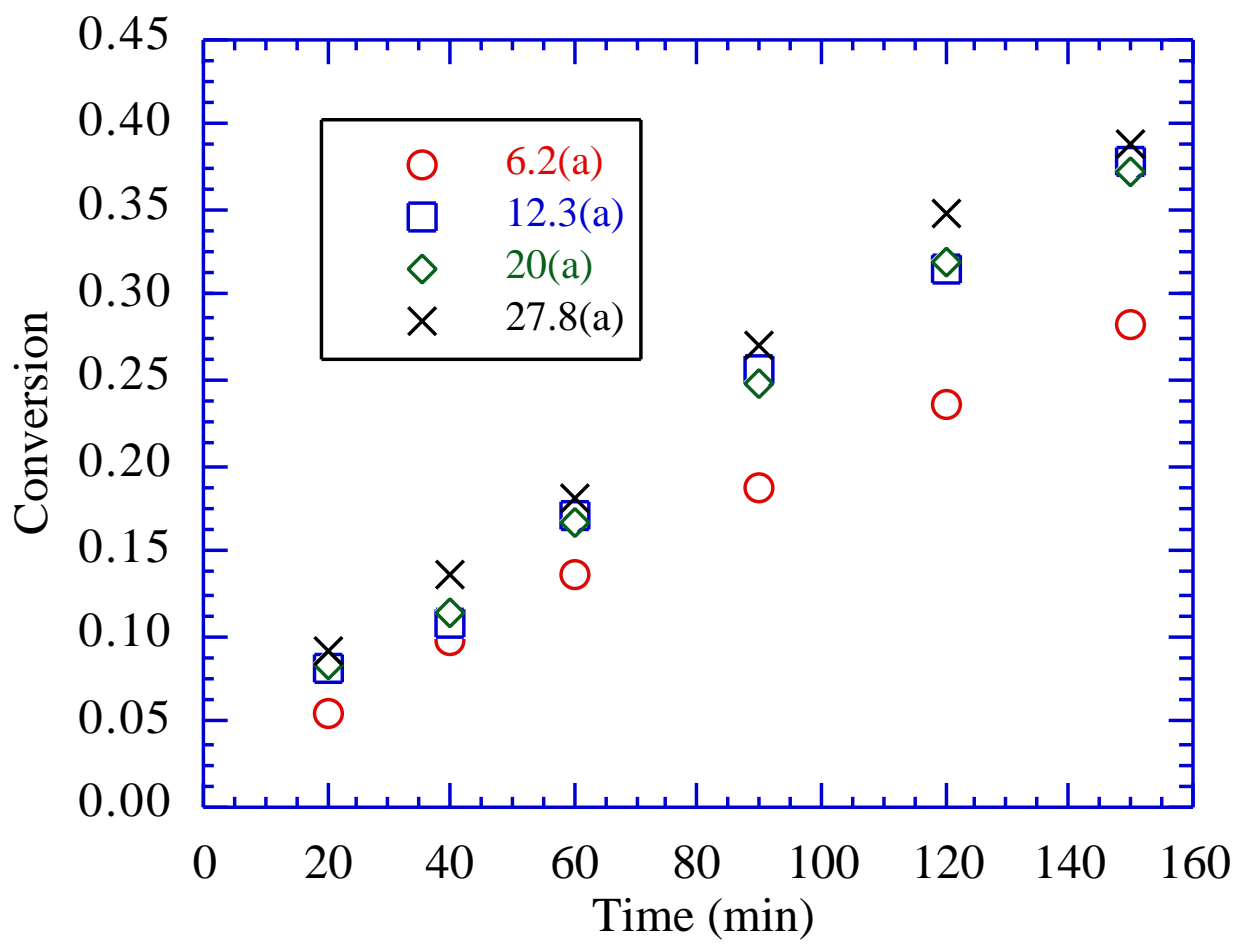

Figure 4: Comparison of neat tetradecane conversions as a function of reactant loading. 
The major products observed from the pyrolysis of tetradecane at all concentrations were $\alpha$-olefins, with minor selectivity to $n$-alkanes. The highest selectivity under all concentrations was obtained for 1-heptene, reaching a maximum of 0.13 for the $5.05 \times 10^{-2} \mathrm{M}$ reactions, and $\alpha$-olefins with carbon numbers of 6 to 11 were also observed in significant quantities. For carbon numbers greater than 8 , the selectivities of $\alpha$-olefins were identical for conversions greater than $\sim 20 \%$. For carbon numbers less than 8 , the product selectivities were maximized for the $20 \mathrm{mg}$ loadings. The selectivity of 1-heptene was similar for the highest loadings, but substantially decreased with lower loadings. Although it is not clear at this point, these observations may be due to partitioning of the higher carbon number products into the liquid phase at higher total pressures. Quantitative analysis of the gaseous products is in progress to investigate this possibility.

The selectivity to $\alpha$-olefins decreased with increasing conversion for all reaction conditions studied. The selectivity for 1 -undecene is plotted in Figure 5 as a representative alkene. The selectivity to n-alkanes significantly increased with both conversion and reactant loading, as shown in Figure 6 for n-heptane as a representative compound in this class. This behavior can be explained by noting that hydrogenation of olefins and continued thermal cracking can occur as both the reaction time and number of intermolecular reactions increase.

The product distributions were rationalized in terms of the typical free radical RiceHerzfeld and Rice-Kossiakoff mechanisms for low pressures, which are represented in Figure 7.13-16 The decomposition is initiated by carbon-carbon bond fission along the main chain to form two primary radicals. These primary radicals form secondary radicals through hydrogen abstraction from a secondary carbon or an intramolecular hydrogen rearrangement. These secondary radicals then undergo $\beta$-scission to form $\alpha$-olefins and primary radicals. Termination occurs by recombination of radicals.

\section{A. 1. B. Neat NBBM Pyrolysis}

Neat pyrolysis of NBBM provided the baseline to which subsequent binary experiments were compared. Two of the major products from pyrolysis of NBBM, which are formed by carbon-carbon bond fission and subsequent hydrogen abstraction, were toluene and 1-methyl-4-(naphthylmethyl) benzene, each observed with a selectivity of greater than 0.28 at all reaction times studied. The other major product, resulting from either hydrogen-carbon bond fission or more likely, intermolecular hydrogen abstraction and a resulting $\beta$-scission, was 1-(2-phenylethenyl)-4-(naphthylmethyl) benzene, with an initial selectivity of approximately 0.35 , which decreased linearly with reaction time to 0.11 at 150 minutes.

Minor selectivities were observed for a number of products from NBBM pyrolysis, the selectivity of which either increased or decreased with increasing reaction time. Methyl bibenzyl and 1-(4-(4-methyl)phenylmethyl)benzyl) naphthalene were observed with initial selectivities of 0.055 and 0.070 , respectively. Each of these showed a reduction in selectivity as reaction time progressed, which is one possible indicator of subsequent decomposition reactions. Other minor products included 1,4-(binaphthylmethyl) benzene, phenyl methyl naphthalene, naphthalene, methyl naphthalene, 1-(phenylmethyl)-4(naphthylmethyl) benzene, 1-methyl-4-(2-phenylethenyl) benzene and p-xylene, which showed increased selectivities.

Mechanistic interpretation using the ideas put forth by Walter et al. successfully accounted for the observed product spectra from neat pyrolysis. The products anticipated from scission of the five main bonds of NBBM, labeled A-E, and subsequent hydrogen 




Figure 5: Comparison of selectivities of 1-undecene as a function of conversion and reactant loading.



Figure 6: Comparison of selectivities for $\mathrm{n}$-heptane as a function of conversion and reactant loading. 
- Initiation

$$
\mathrm{R}_{\mathrm{k}} \stackrel{\mathrm{k}_{1}}{\longrightarrow} \mathrm{R}_{\mathrm{i}}-\mathrm{CH}_{2}-\mathrm{CH}_{2}+\mathrm{R}_{\mathrm{j}}
$$

- Propagation

$$
\begin{aligned}
& \cdot \mathrm{R}_{\mathrm{j}}+\mathrm{R}_{\mathrm{k}} \stackrel{\mathrm{k}_{2}}{\longrightarrow} \mathrm{R}_{\mathrm{j}}+\mathrm{R}_{\mathrm{i}}-\dot{\mathrm{C}} \mathrm{H}-\mathrm{CH}_{2}-\mathrm{R}_{\mathrm{j}} \\
& \mathrm{R}_{\mathrm{i}} \longrightarrow \stackrel{\dot{\mathrm{C}} \mathrm{H}}{-} \mathrm{CH}_{2}-\mathrm{R}_{\mathrm{j}} \stackrel{\mathrm{k}_{3}}{\longrightarrow} \mathrm{R}_{\mathrm{i}}-\mathrm{CH}=\mathrm{CH}_{2}+\cdot \mathrm{R}_{\mathrm{j}}
\end{aligned}
$$

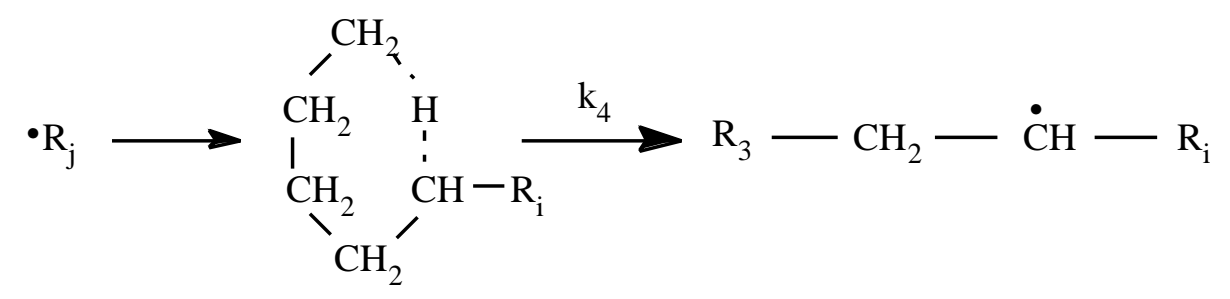

- Termination

$$
\begin{aligned}
& \cdot \mathrm{R}_{\mathrm{j}}+\cdot \mathrm{R}_{\mathrm{j}} \stackrel{\mathrm{k}_{5} \longrightarrow}{\longrightarrow} \\
& \cdot \mathrm{R}_{\mathrm{j}}+\mathrm{R}_{\mathrm{i}}-\dot{\mathrm{C}} \mathrm{H}-\mathrm{CH}_{2}-\mathrm{R}_{\mathrm{j}} \stackrel{\mathrm{k}_{6} \longrightarrow}{\longrightarrow} \\
& \mathrm{R}_{\mathrm{i}} \stackrel{\mathrm{C}}{\longrightarrow}-\mathrm{CH}_{2}-\mathrm{R}_{\mathrm{j}} \stackrel{\mathrm{k}_{7}}{\longrightarrow} \\
& \mathrm{R}_{\mathrm{i}} \longrightarrow \mathrm{C}_{\mathrm{j}} \stackrel{\mathrm{CH}_{2}-}{\longrightarrow}
\end{aligned}
$$

Figure 7: Proposed mechanism for pyrolysis of tetradecane (Rice and Herzfeld, 1933; Kossiakoff and Rice, 1943). 
abstraction and $\beta$-scission, are depicted in Figure 8. The formation of high yields of toluene and 1-methyl-4-(naphthylmethyl) benzene is consistent with the proposed mechanism involving bond D fission. This is the weakest bond in the molecule, since the radicals which are formed can be stabilized by the adjacent benzyl substituents. Similarly, the $\mathrm{C}_{2}$ linkage in NBBM possesses the most easily abstractable hydrogens. Once a secondary radical is formed, it can undergo $\beta$-scission to form 1-(2-phenylethenyl)-4(naphthylmethyl) benzene. This compound could then undergo degradation reactions similar to those observed for NBBM, leading to a reduction in selectivity as reaction time increases.

A comparison of the selectivities of products associated with direct cleavage of bond $\mathrm{A}$ as a function of conversion is shown in Figure 9. If these products were solely formed by bond A scission, it would be expected that the selectivities would be equal for naphthalene and the sum of methyl bibenzyl and its corresponding derivatives. This is clearly not the case. Therefore, another reaction pathway for the formation of these products must exist.

Upon examination of other bond scission pairs, a discrepancy between bond $\mathrm{C}$ products, phenyl methyl naphthalene, which was observed, and ethylbenzene, which was not, was noted. Also, as stated, other products which can not be explained by one of the five bond scissions were observed, which leads to investigation of secondary pathways. These observations are consistent with a free radical ipso-substitution scheme for the formation of the various products as proposed by Walter et al. For example, attack by a benzyl radical of the NBBM molecule at the 1-naphthyl position would afford phenyl methyl naphthalene. This scheme would involve the formation of a mole of naphthalene and phenyl methyl naphthalene for every mole formed of methyl bibenzyl and its derivatives. This comparison is presented in the plot of Figure 10. Likewise, various radical attacks at bond $C$ can explain the appearance of 1-(4-(4methyl)phenylmethyl)benzyl) naphthalene, 1,4-(binaphthylmethyl) benzene, and 1-(phenylmethyl)-4-(naphthylmethyl) benzene. Overall, the main reaction families for NBBM pyrolysis are therefore bond thermolysis, hydrogen abstraction, radical ipso-substitution, $\beta$-scission, and radical recombination. ${ }^{12}$

\section{A. 1. C. Binary Mixture Pyrolysis}

Reactions of binary mixtures of tetradecane and NBBM revealed interactions between the reactants and synergistic effects. As observed in Figure 11, the conversion of tetradecane was significantly enhanced for all of the reactions which were conducted in the presence of NBBM. The pseudo-first order rate constants for tetradecane degradation increased from $2.17 \times 10^{-3} \mathrm{~s}^{-1}$ to $3.78-4.15 \times 10^{-3} \mathrm{~s}^{-1}$ for the $6.2 \mathrm{mg}$ loadings, and $3.13 \times 10^{-3} \mathrm{~s}^{-1}$ to $5.15 \times 10^{-3} \mathrm{~s}^{-1}$ for the $12.3 \mathrm{mg}$ loading. These increases were rationalized in terms of kinetic coupling. ${ }^{17}$ The internal carbon-carbon bonds of tetradecane have a higher bond dissociation energy $\left(90 \mathrm{kcal} \mathrm{mol}^{-1}\right)$ than that of bond D in NBBM $\left(60 \mathrm{kcal} \mathrm{mol}^{-1}\right) .18$ This has the potential to increase the quantity of radicals in the system with respect to the neat tetradecane experiments at a particular reaction time. The NBBMderived radicals can easily abstract hydrogen from the secondary carbons of tetradecane, forming a tetradecane-derived radical and converting a tetradecane molecule, enhancing its conversion. Once formed, these tetradecane-derived radicals undergo their own decomposition reactions as observed for neat pyrolysis.

The interactions between NBBM and tetradecane can be further clarified by examining the products derived from NBBM. Since abstraction of hydrogen from tetradecane is facile and has a high reaction path degeneracy of $24, \mathrm{NBBM}$ radicals are 


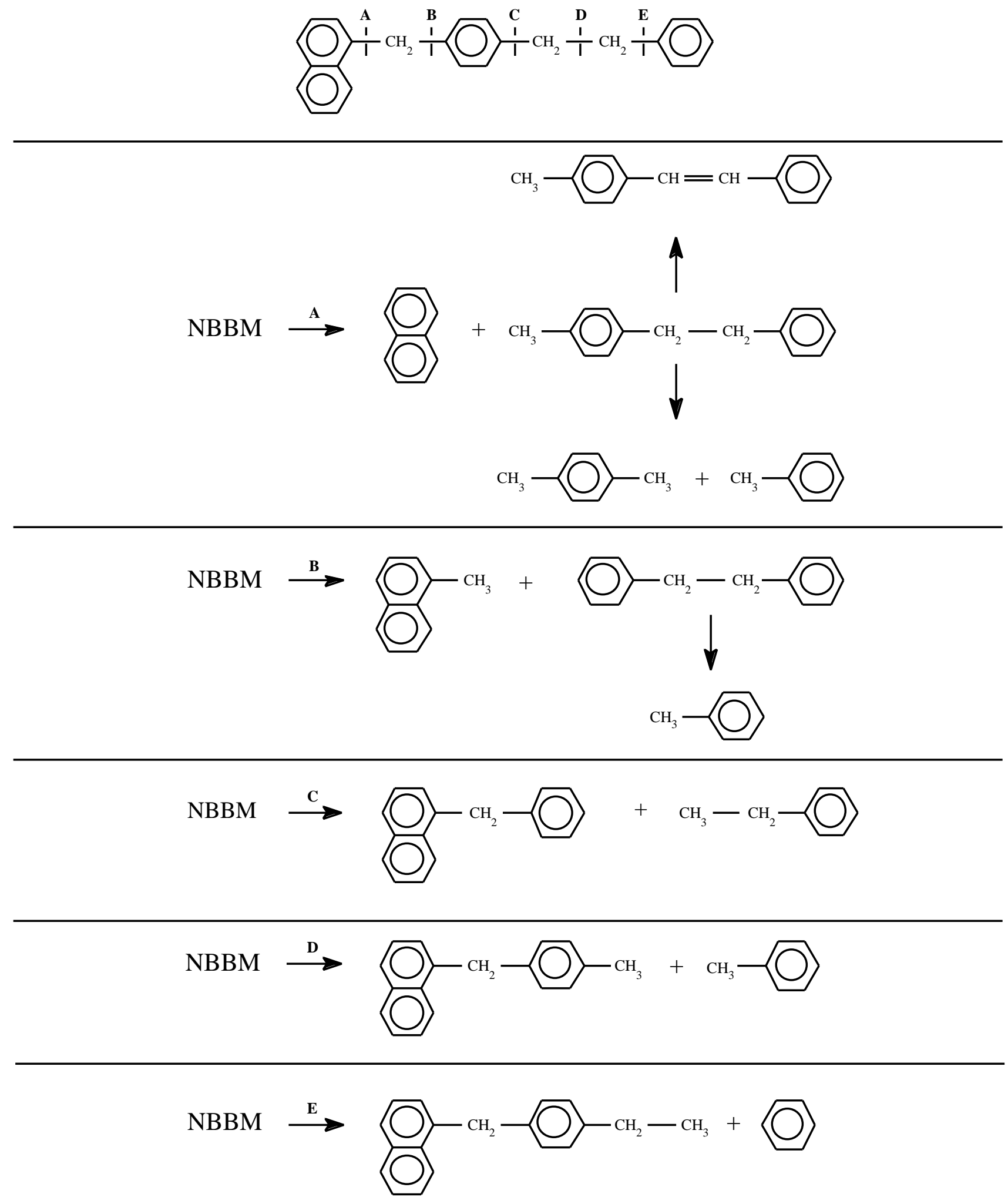

Figure 8: Bond Assignment and corresponding products of NBBM pyrolysis, allowing for only bond fission, hydrogen abstraction, and $\beta$-scission. 


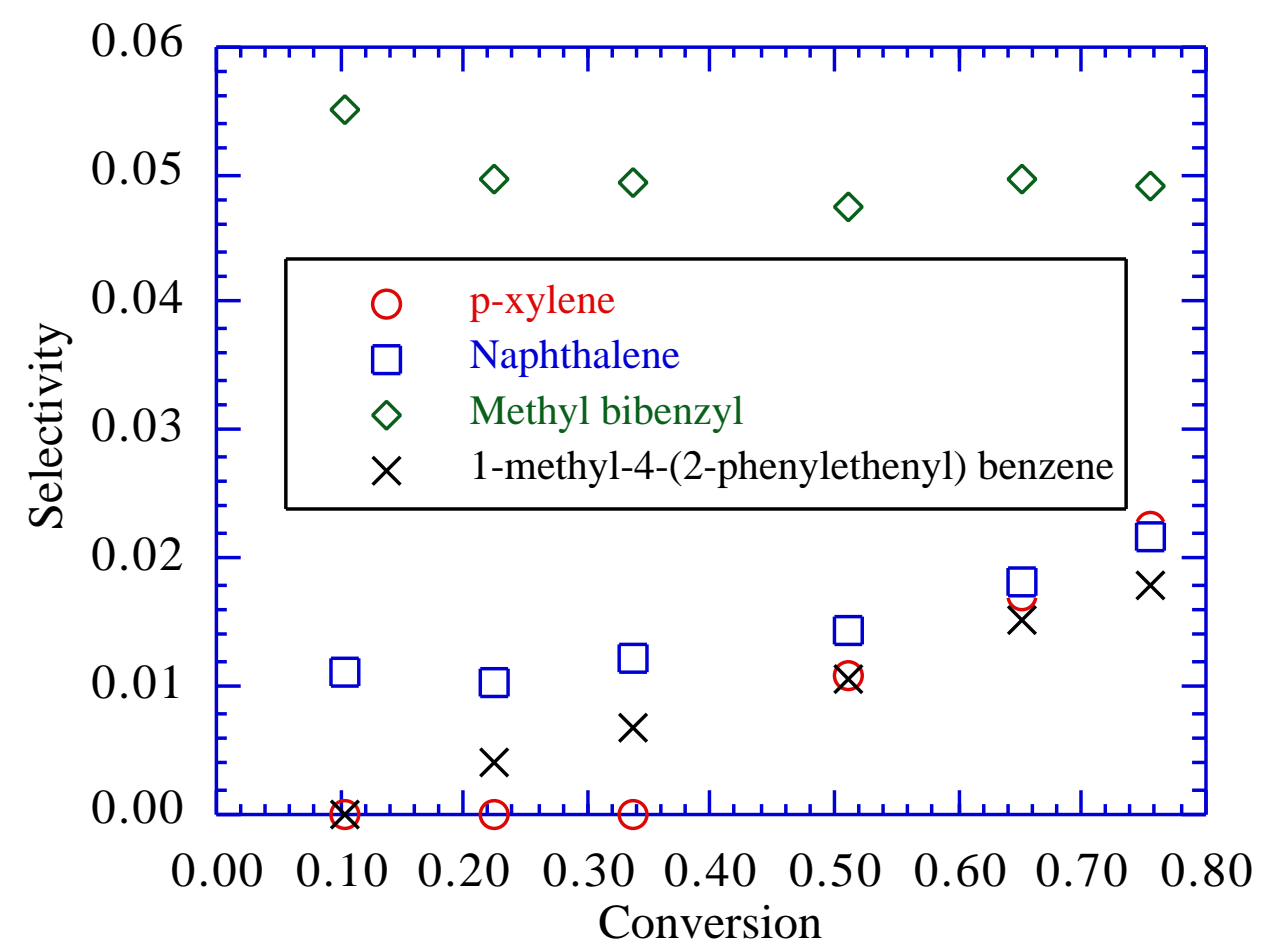

Figure 9: Comparison of selectivities of products associated with direct cleavage of bond $\mathrm{A}$ as a function of conversion.

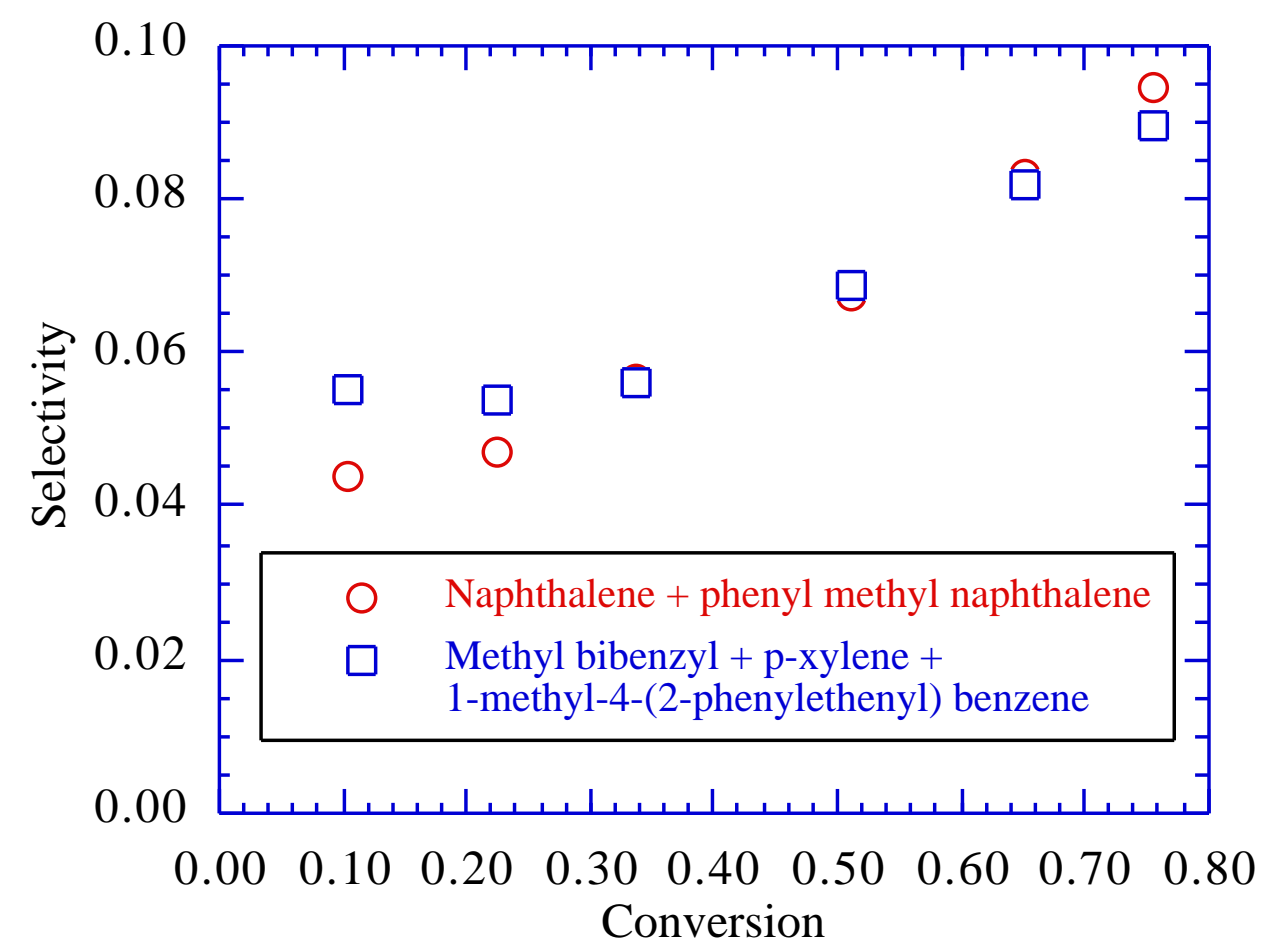

Figure 10: Comparison of products from free radical ipso-substitution reaction which accounts for cleavage of bond A. 


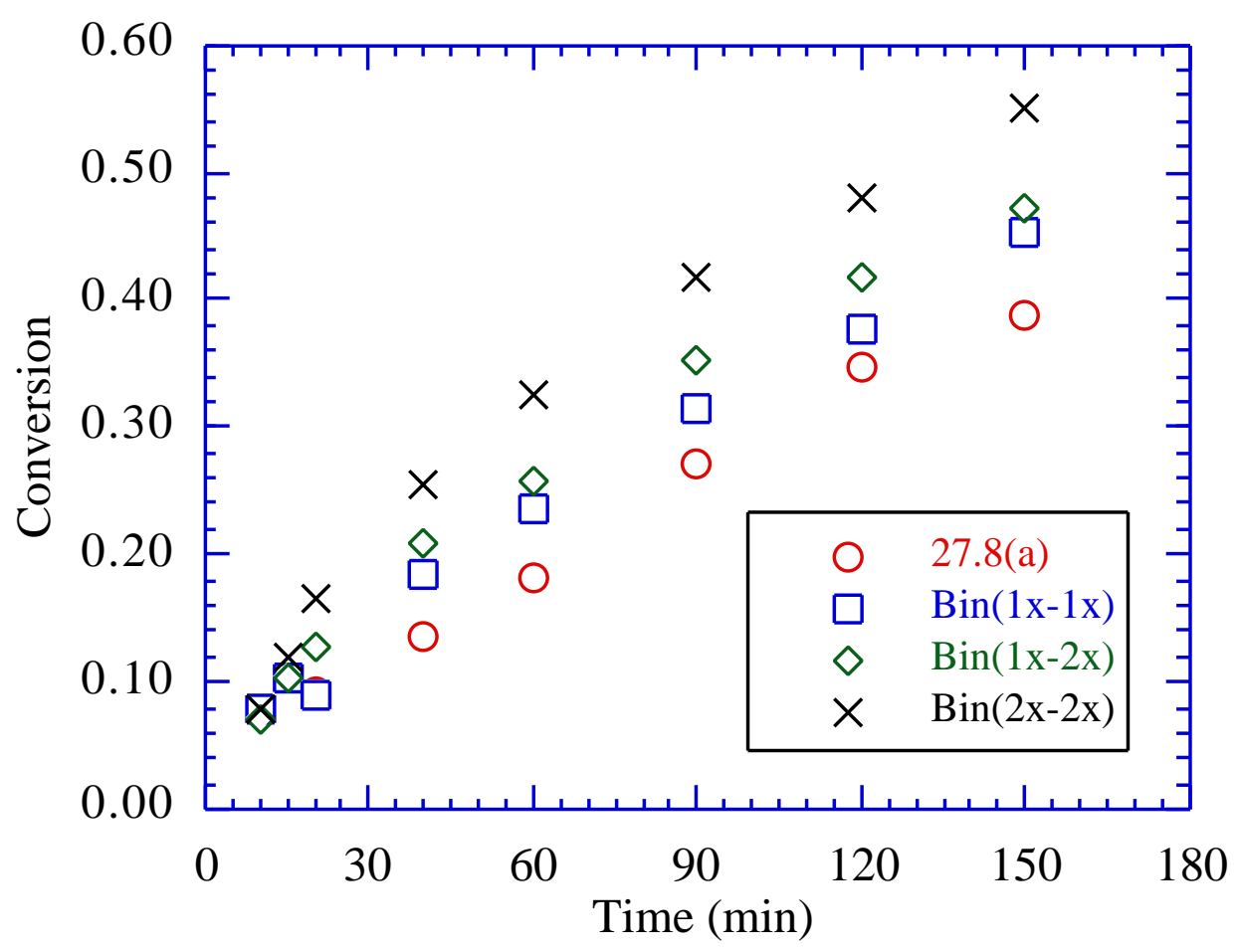

Figure 11: Comparison of tetradecane conversions as a function of reactant loading during binary reactions.

capped and stable before undergoing secondary reactions. This effect on the overall product yields can be discerned from Figures 12 and 13. The radicals formed from bond D thermolysis abstract hydrogen with higher selectivity and afford higher yields of toluene and its compliment. Correspondingly, the selectivity to the radical ipso-substitution pathway and formation of phenyl methyl naphthalene is diminished.

Both the initial charge of each reactant and the overall reactant loading strongly influenced reactant conversions and product selectivities during coprocessing. The conversion of tetradecane was most dramatically affected by both of these factors during the binary experiments. In order to quantify the effect of increasing the initial NBBM to tetradecane ratio, the $\operatorname{Bin}(1 \mathrm{x}-1 \mathrm{x})$ and $\operatorname{Bin}(1 \mathrm{x}-2 \mathrm{x})$ reactions were compared. As the loading of NBBM was increased, the rate of conversion of tetradecane was enhanced. This result is consistent with an increase in the effective radical concentration in the system with a higher loading of NBBM, which in turn increases the conversion of the polymer mimic. The rate enhancement observed during neat pyrolysis for tetradecane as the loading was increased is also observed when NBBM is present. The rate of conversion of tetradecane was enhanced at a constant NBBM loading, as the rate was higher for the $\operatorname{Bin}(2 x-2 x)$ than for $\operatorname{Bin}(1 \mathrm{x}-2 \mathrm{x})$. However, a reduction in conversion was observed at long reaction times. The highest rate of conversion was obtained when both factors contributed to the disappearance of tetradecane, as the $\operatorname{Bin}(2 \mathrm{x}-2 \mathrm{x})$ showed the largest rate of conversion. As the total reactant concentration increases, the rate of bimolecular reactions increases. The NBBM-derived radicals selectively abstract hydrogen from the tetradecane chains with an even higher rate with increasing concentrations, thus increasing the rate of tetradecane degradation further. Therefore, increasing both the molar ratio of NBBM to tetradecane and the total molar concentration in the system results in an enhancement of the polymer model compound reactivity. 


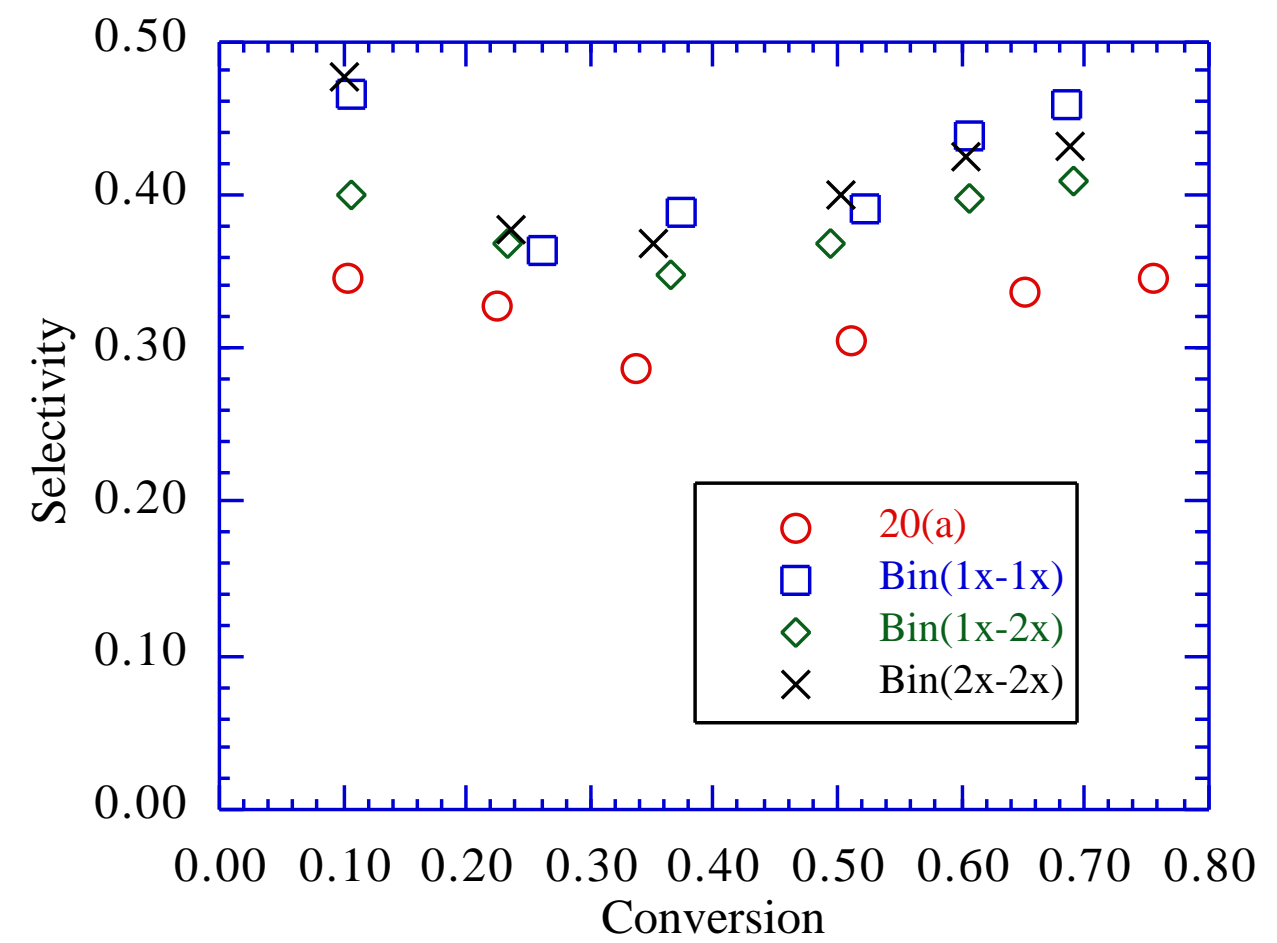

Figure 12: Comparison of toluene selectivity as a function of reactant loading.

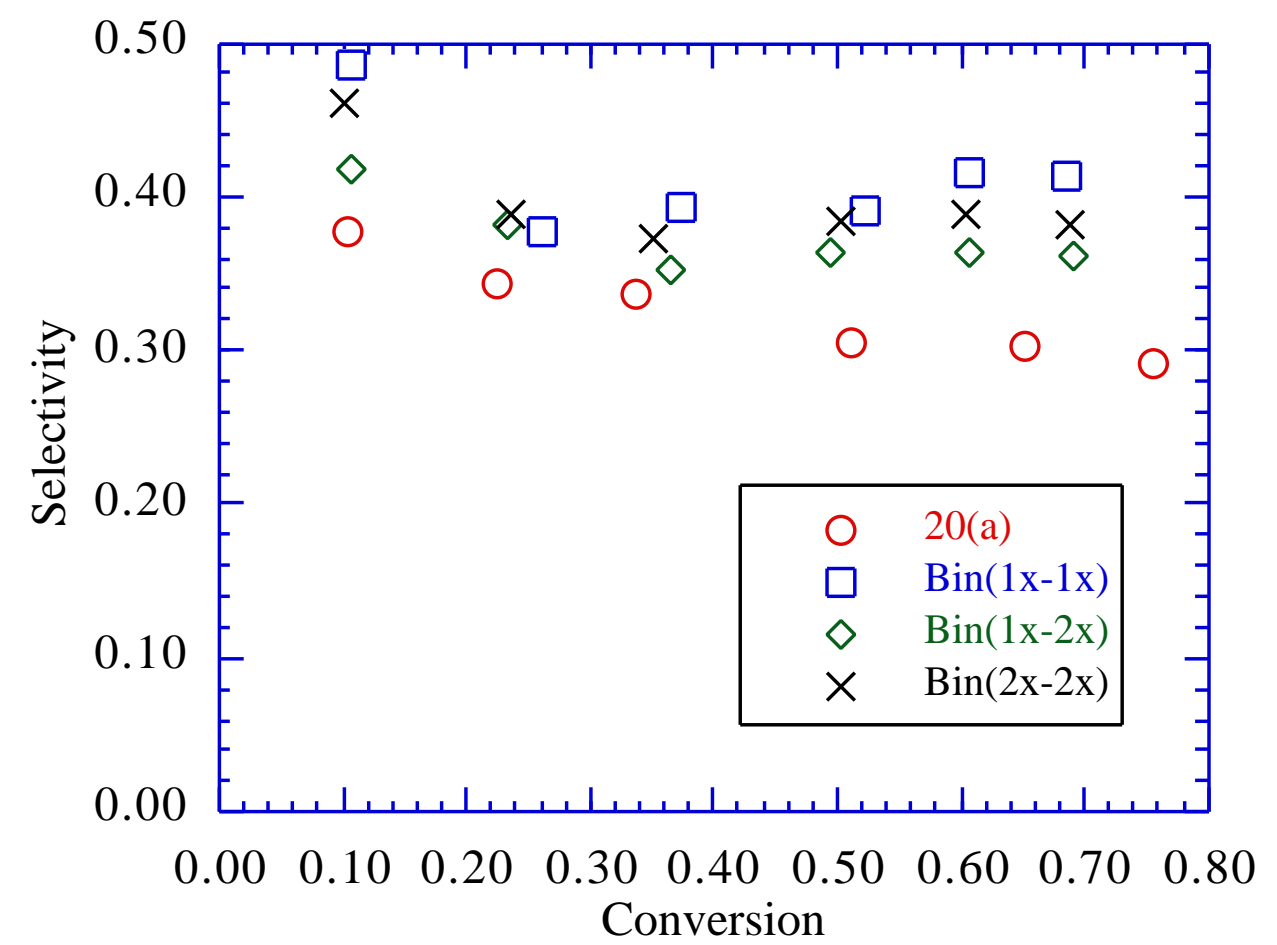

Figure 13: Comparison of 1-methyl-4-(naphthylmethyl) benzene as a function of reactant loading. 
Although changes in concentration also influenced the major products of NBBM, the conversion of NBBM was not strongly affected. As shown in Figure 14, the conversion of NBBM was slightly enhanced at low conversions during binary reactions. As discussed previously, the degradation of the coal model compound primarily occurs through the thermolysis of bond $D$. The radicals afforded will react selectively with tetradecane, rather than with other NBBM molecules. Therefore, at low times, the conversion will only be minimally affected and will be very similar to the neat reactions. At long reaction times, higher conversion will be attained for neat pyrolysis due to secondary reactions between NBBM-radicals and NBBM.

The selectivities of the primary products of NBBM were strongly affected by changes in reactant concentrations. As the overall reactant loadings and NBBM to tetradecane ratios were raised, the selectivity to toluene and its compliment decreased as shown in Figures 12 and 13. These observations are consistent with the mechanistic scenario used to explain the changes observed in reactant conversions. The lowest binary loadings showed the highest selectivities, which implies that NBBM radicals which are formed are stabilized by tetradecane and are less likely to undergo further secondary and tertiary reactions. As the NBBM to tetradecane ratio increased, however, the NBBM radicals have a higher probability of reacting with other NBBM-derived species, thus resulting in a decrease of primary products. Similarly, by increasing the overall reactant concentration, the quantity of bimolecular reactions increased, resulting in a higher rate of reaction of NBBM and its products. However, the effect of increasing the molar ratio dominates the effect of the overall reactant loading on the selectivity to primary products during coprocessing of polymer mimics with coal model compounds. This is illustrated by the trends observed for the selectivity of phenyl methyl naphthalene, a major product formed through secondary reactions, which are shown in Figure 15.

It has been shown that during low pressure coprocessing of coal and polymer model compounds, favorable interactions between reactants exist. In binary mixtures, the conversion of tetradecane increased while the selectivity to primary products of NBBM pyrolysis was enhanced. These observations were attributed to the stabilization of NBBMderived radicals through hydrogen abstraction from tetradecane which in turn, increases the rate of tetradecane conversion. The relative concentrations of each reactant also affected the tetradecane conversion and selectivity towards primary products of NBBM. Increasing both the NBBM to tetradecane ratios and the overall reactant loading resulted in an increase of interactions between NBBM and its derivatives and an enhancement of tetradecane degradation.

\section{A. 2. High Pressure Reactions}

Preliminary high pressure experiments were conducted using tetradecane. In order to compare directly the results of these experiments to those obtained from the low pressure reactions, reactant concentrations equal to the low pressure reactions were employed. For the first set of high pressure experiments, the $12 \mathrm{cc}$ stainless steel reactors were filled with ca. $120 \mathrm{mg}$ of tetradecane $\left(5.05 \times 10^{-2} \mathrm{M}\right)$ and pressurized to 1000 psig (cold) with nitrogen. Thermal degradation was conducted at $420^{\circ} \mathrm{C}$ for $20-150$ minutes.

These experiments initially yielded a similar product spectra to the low pressure neat pyrolysis reactions at similar concentration. The primary products in the liquid fraction were $\alpha$-olefins and n-alkanes, but the $\alpha$-olefins to n-alkane ratio was lower than that obtained during the low pressure runs. This is consistent with results of long chain paraffin cracking conducted at elevated pressures reported in the literature. ${ }^{16,18-20}$ At the high pressures and moderate temperatures employed in this study, the frequency of 


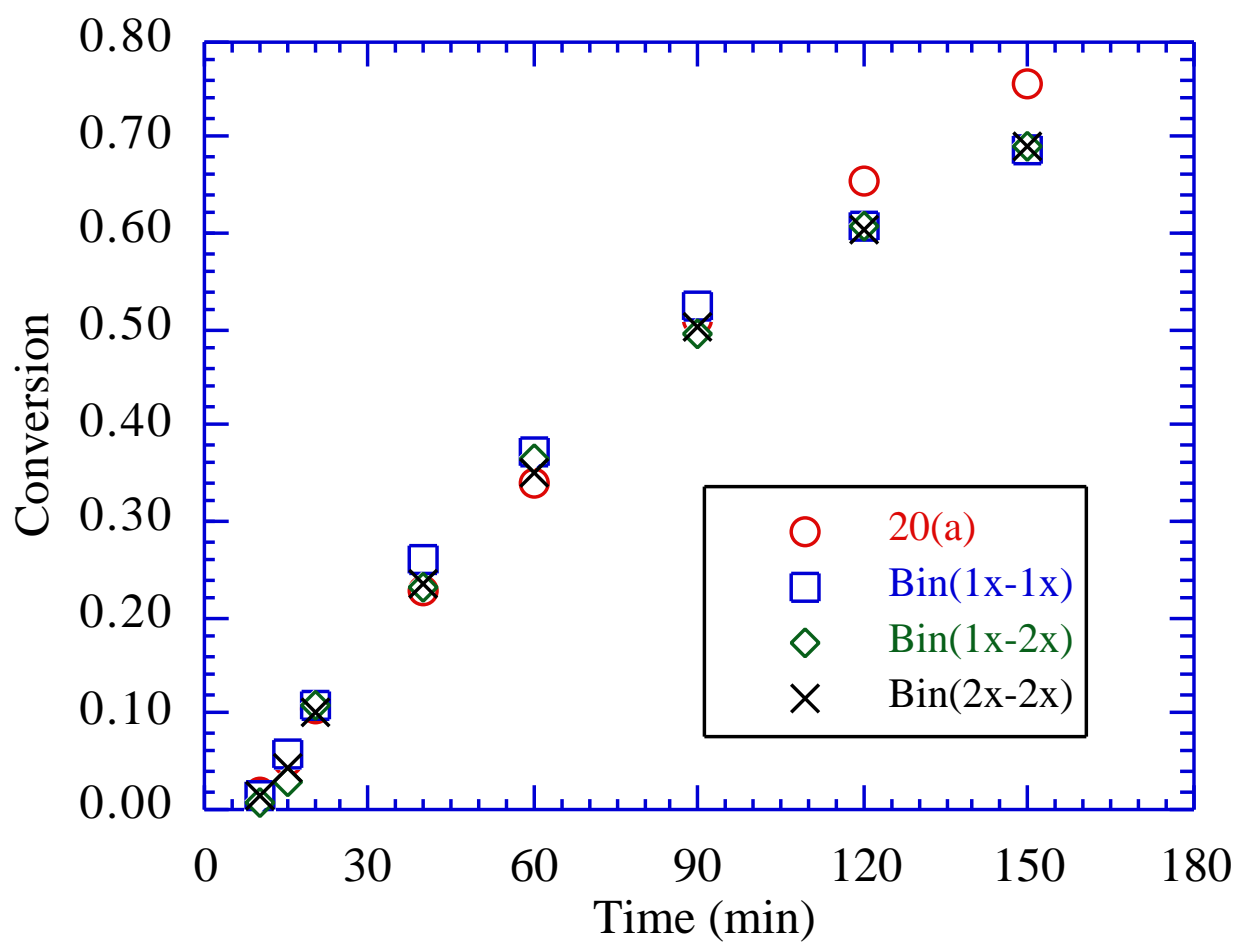

Figure 14: Comparison of conversion of NBBM as a function of reactant loading.

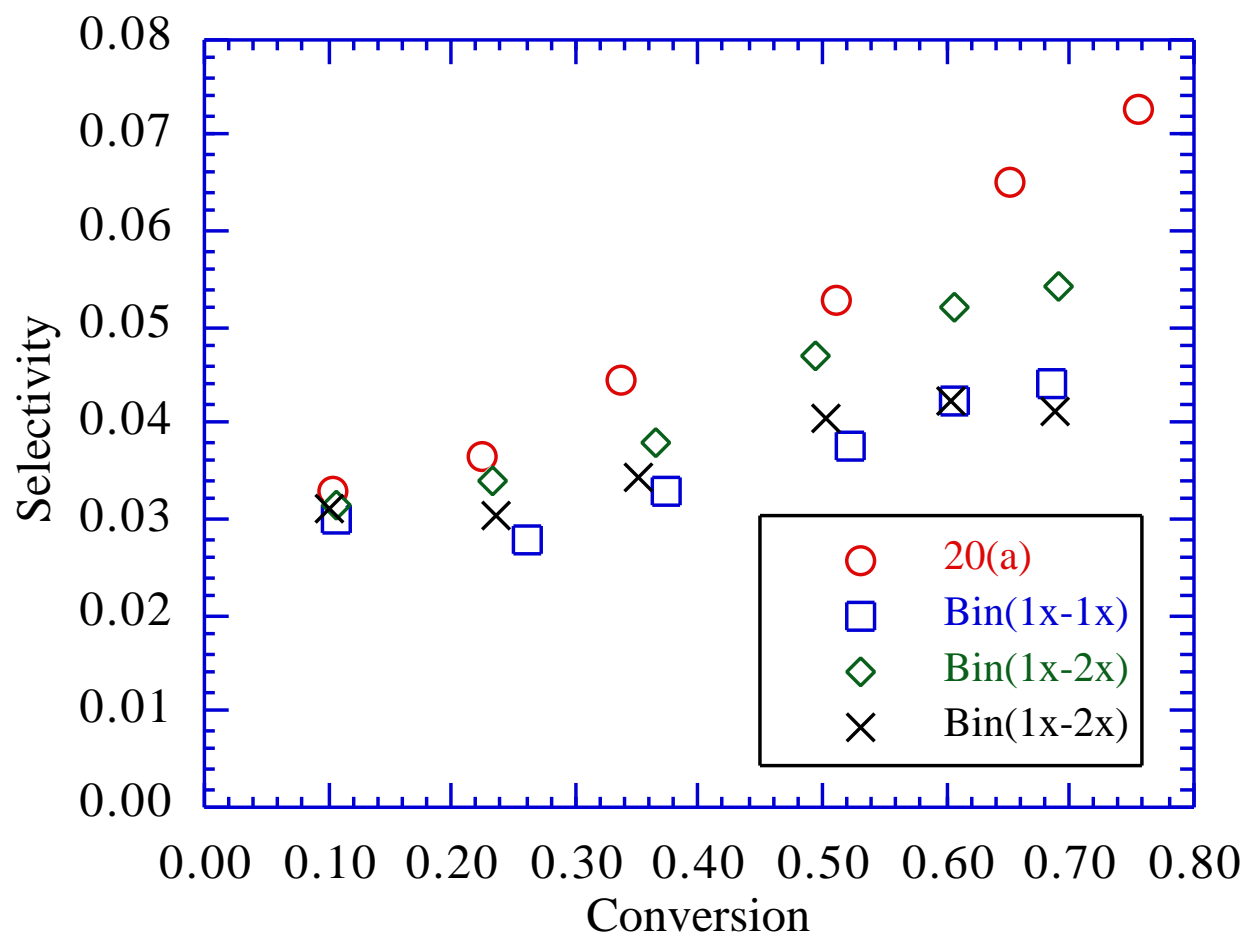

Figure 15: Comparison of selectivities of phenyl methyl naphthalene as a function of reactant loading and conversion. 
bimolecular reactions is enhanced, with hydrogen abstraction becoming comparable to $\beta$-scission. ${ }^{18,20}$ This will lead to an increase in n-alkanes as compared to the low pressure pyrolysis, yielding lower olefin to alkane ratios.

The types of gaseous products were similar to those in the liquid fraction, primarily consisting of n-alkanes and olefins. However, the olefin to $n$-alkane ratio for $C_{2}-C_{4}$ was less than 1. These smaller species may be hydrogenated very readily through intermolecular reactions, resulting in significantly lower olefin to alkane ratios. ${ }^{20}$ The formation of these products occurs through mechanisms similar to those for higher carbon numbers, but $\mathrm{C}_{2}$ products can also be formed from $\beta$-scission of a primary radical of any length greater than 2 . This will yield a high proportion of $\mathrm{C}_{2}$ products during pyrolysis.

As additional runs were carried out, the reactant conversions obtained during the high pressure reactions were very inconsistent. There was an increase in conversion for a particular reaction time for subsequent runs. The higher conversions gave rise to higher proportions of n-alkanes and the appearance of aromatics and naphthenes, as anticipated from the higher conversion data obtained at low pressure and $500^{\circ} \mathrm{C}$. These products would result from continued bimolecular, condensation (e.g. Diels-Alder), and ring-closure reactions. Though these reaction mechanisms are consistent with pyrolysis of linear hydrocarbons at high conversions, it was hypothesized that the inconsistencies in the reactant conversion could possibly be attributed to catalytic wall effects within the reactor. Although stainless steel does not typically develop catalytic effects below $550^{\circ} \mathrm{C}$, it is possible that such effects could be significant. ${ }^{16,21}$

In order to determine the extent of the possible catalytic effects, further experiments are required. The reactors may possibly be undergoing a conditioning period, after which consistent and accurate results will be obtained. However, the catalytic activity may possibly persist if it is due to factors other than a conditioning period. Therefore, glass sleeves have been fabricated which will be placed in the reactors to minimize wall effects. Experiments will be conducted using the sleeves in order to determine if the reactions are being conducted without catalytic enhancement. Once catalytic effects have been eliminated, high pressure reactions will be conducted which can then be compared to the low pressure experiments. Following these experiments, the effects of adding hydrogen, solvents, and catalysts to the system will be addressed.

\section{B. Real Feedstock Studies}

Three sets of pyrolysis experiments, using loadings of $12.3,20.0$, and $27.8 \mathrm{mg}$ respectively, were performed in $2 \mathrm{ml}$ glass ampoules with $\mathrm{HDPE}$ at $420^{\circ} \mathrm{C}$ for $30-180$ minutes. At the end of the reaction period, the ampoules were placed in a room temperature fluidized sand bath to cool. Product identification and quantification were conducted using the methods described in the experimental section of this report.

Reactant conversions were calculated as described earlier using weights of extracted products. An alternative measure of reactant conversion was obtained by summing the quantified individual product yields and dividing by the initial reactant loading. There were significant differences between the two calculated values for conversion, with the values obtained from solvent extraction yielding conversions greater than $100 \%$. The conversions obtained by summing product yields resulted in much more reasonable values and were in much better agreement with one another. These values also seemed reasonable when coupled with the known amount of wax remaining in the ampoules after extraction. In order to compare specific reaction products directly, normalized molar yields, defined as 
the number of moles of product $\mathrm{i}$ divided by the corresponding reactant charge, were calculated and plotted as a function of reaction time.

The primary products observed during HDPE pyrolysis were linear hydrocarbons, consisting of $\alpha$-olefins, n-alkanes, and internal olefins, which is consistent with results from the literature. ${ }^{22}$ These products are similar to those found during tetradecane pyrolysis, indicating that both feedstocks decompose by the same basic mechanisms. The $\alpha$-olefins were initially found in the highest yield for all reaction sets, and increased with higher conversions. The yields of paraffins increased significantly with longer reaction times, while internal olefin yields also increased, but at a slower rate. Initially, the overall yields of $\alpha$-olefins for carbon numbers $\mathrm{C}_{8}-\mathrm{C}_{24}$ were very similar. At longer reaction times, however, there was an increased selectivity towards products of smaller carbon numbers. These trends are shown in Figures 16 and 17 for the $20 \mathrm{mg}$ reactions. The selectivity increase occurs because shorter hydrocarbon chains are more stable than longer ones. There is a lower probability that shorter chains will further decompose due to the reduction in the number of possible sites for main chain cleavage.

The n-alkanes showed an increased selectivity towards smaller products, but there was also a significant increase in yields of longer chain hydrocarbons. In particular, the yields of n-alkanes of carbon numbers $\mathrm{C}_{20}-\mathrm{C}_{24}$ showed significant growth with reaction time, which is contrary to the behavior of the $\alpha$-olefins of the same carbon numbers. These observations are shown in Figures 18 and 19 for the $20 \mathrm{mg}$ experiments. The increase in the yields occurs since n-alkanes can be formed not only through cleaving and coupling reactions, but also through hydrogenation of $\alpha$-olefins of the same length. As a result of these effects, the $\alpha$-olefin to n-alkane ratio became smaller with increasing reaction time for carbon numbers greater than $\sim 12$.

The significant yields of internal olefins during HDPE pyrolysis were most likely due to bimolecular reactions through hydrogen abstraction by a radical to form a secondary radical. ${ }^{20}$ However, the yields of internal olefins were lower than either the n-alkanes or the terminal olefins.

As was observed during tetradecane pyrolysis, the concentration of reactant in the system plays a role in determining the final product distribution during thermal degradation of HDPE. The yields of low carbon number $\alpha$-olefins were similar for the three loadings at low reaction times, but begin to deviate at longer times. The longer chains, however, showed significant deviation in terminal olefin yields over the full range of reaction times studied. As observed in Figure 20, the highest yields for tetracosene $\left(\mathrm{C}_{24} \mathrm{H}_{48}\right)$ occurred during the 12.3 loading experiments, with decreasing yields for increasing loadings. The yields of n-alkanes for higher carbon numbers were found to show the opposite trend from the $\alpha$-olefins, increasing with larger reactant loads. These observations can be attributed to a larger amount of intermolecular reactions in the gas phase due to higher concentrations, as observed during neat tetradecane pyrolysis. For both sets of thermal degradation experiments, the increase in bimolecular reactions results in higher yields of n-alkanes, partially at the expense of $\alpha$-olefins. This effect is more notable for the HDPE reactions, though, since there is a much larger range of hydrocarbon species within the system. As a result, the $\alpha$-olefin to $n$-alkane ratio was found to decrease with increasing reactant loading for all carbon numbers. This trend is shown in Figure 21 for the ratio of octadecene $\left(\mathrm{C}_{18} \mathrm{H}_{36}\right)$ to octadecane $\left(\mathrm{C}_{18} \mathrm{H}_{38}\right)$.

The trends in the product ratios are similar to those found during tetradecane pyrolysis, but the changes are more marked for the HDPE reactions. The magnitudes of the changes in product ratios were significantly higher for pyrolysis of the polymer than for 


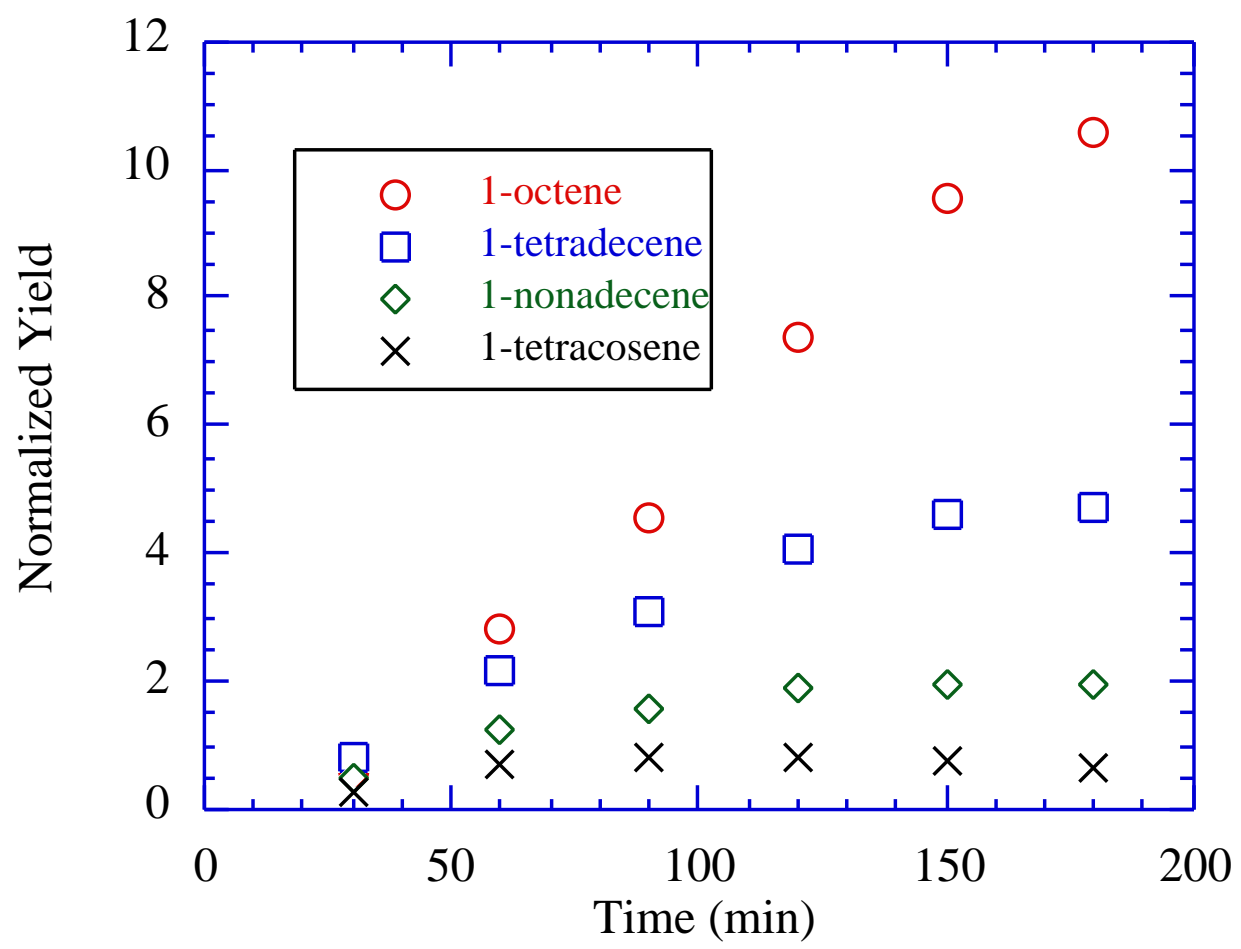

Figure 16: Comparison of normalized yields of selected $\alpha$-olefins for $20 \mathrm{mg}$ reactant loading of HDPE.

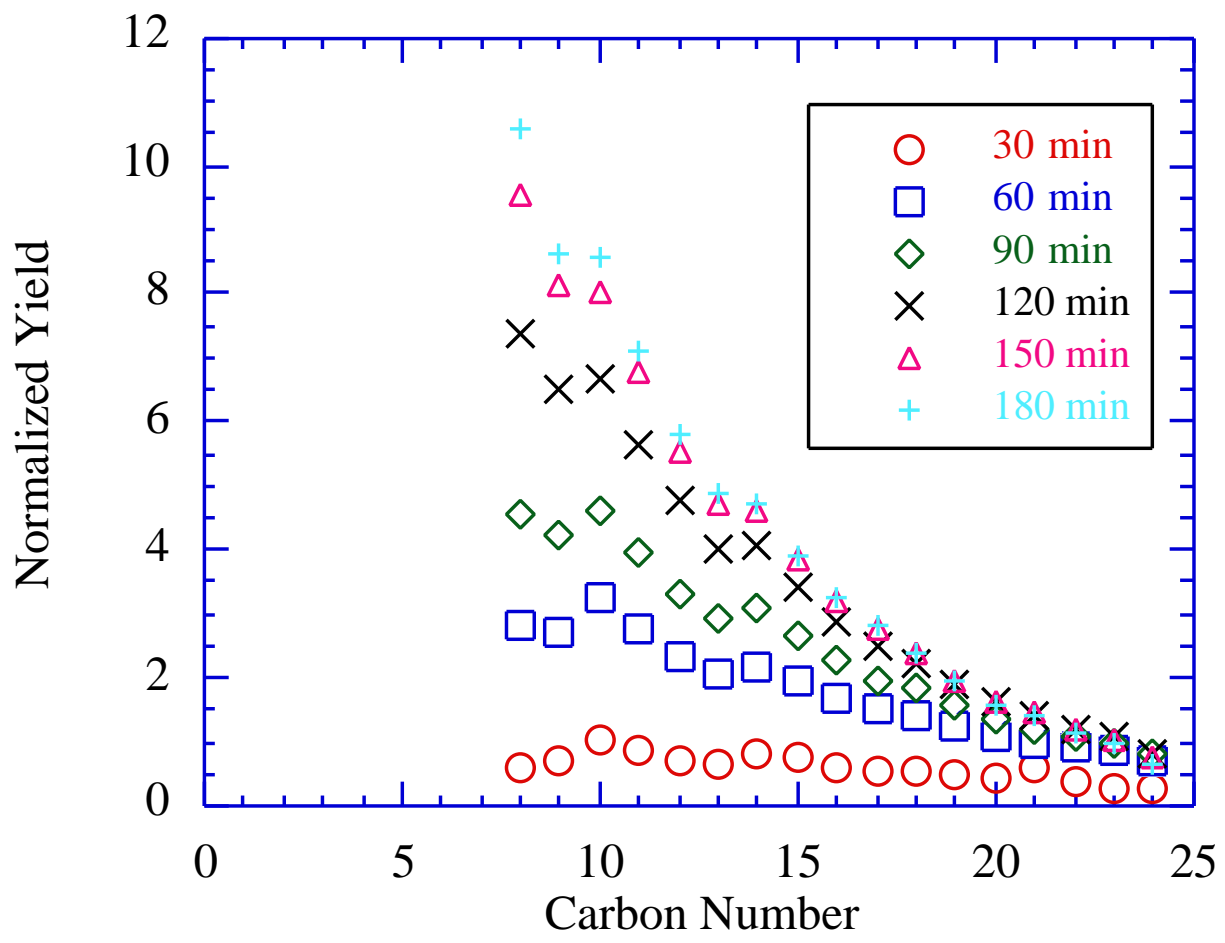

Figure 17: Comparison of normalized yields of $\alpha$-olefins as a function of time and carbon number for $20 \mathrm{mg}$ reactant loading of HDPE. 


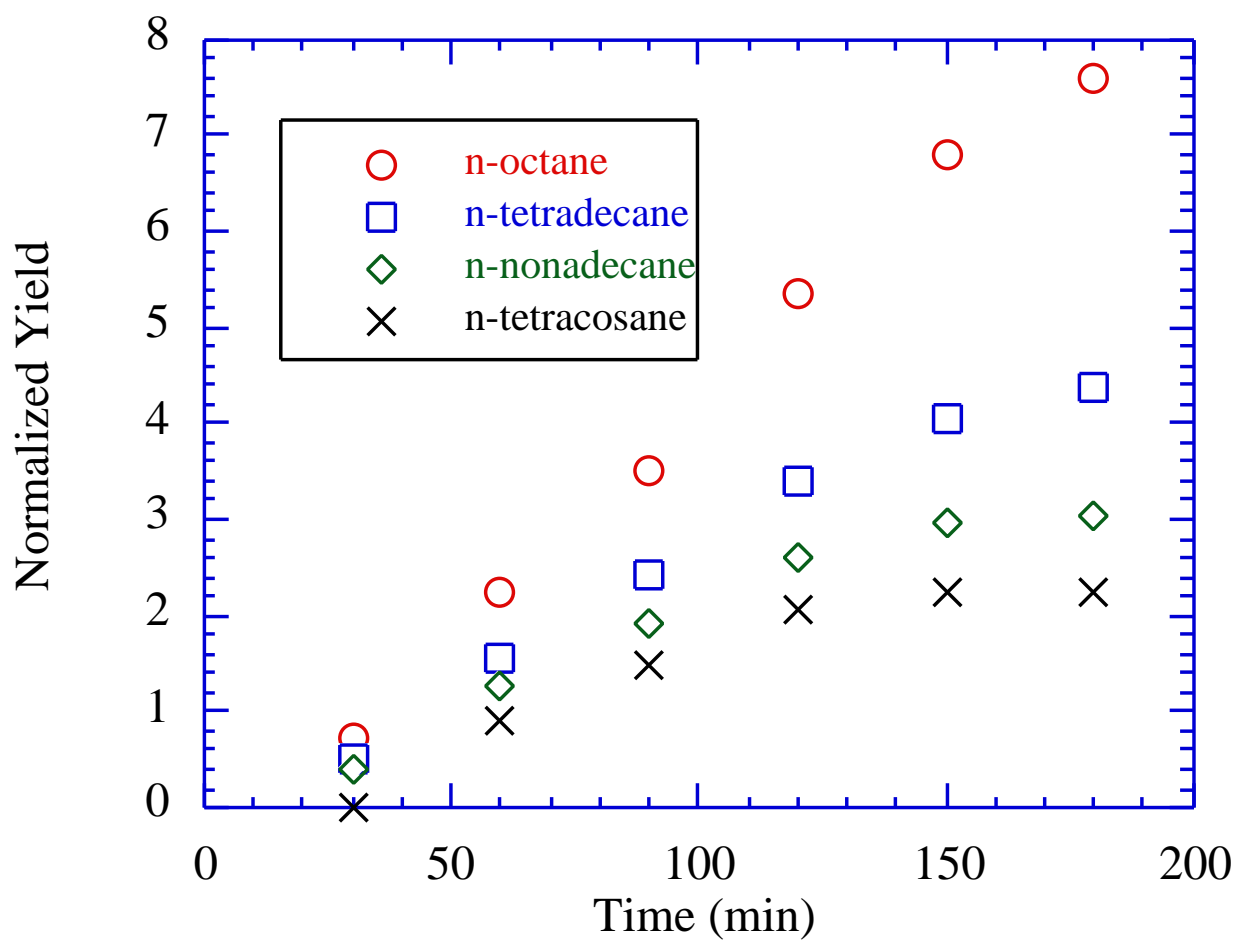

Figure 18: Comparison of normalized yields of selected n-alkanes for $20 \mathrm{mg}$ reactant loading of HDPE.

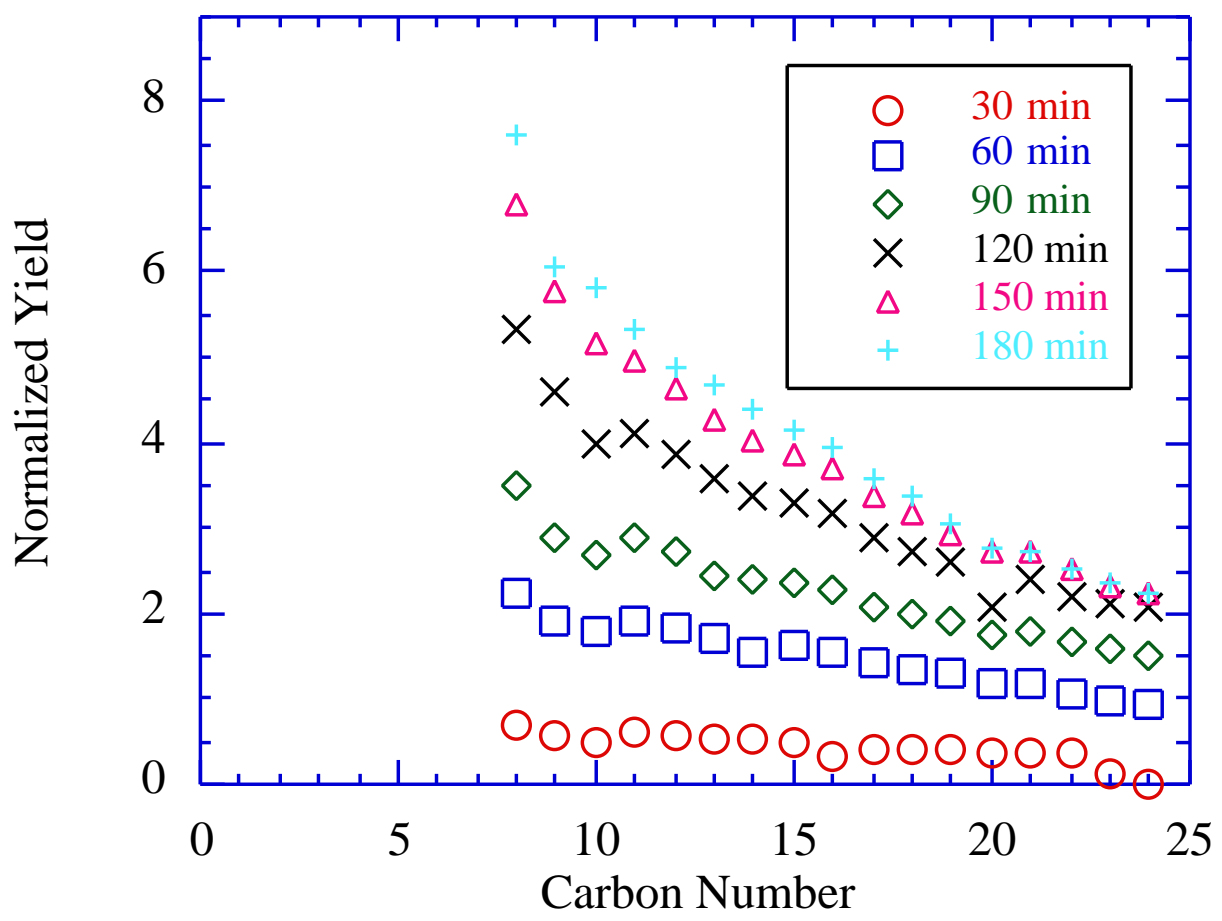

Figure 19: Comparison of normalized yields of n-alkanes as a function of time and carbon number for $20 \mathrm{mg}$ reactant loading of HDPE. 


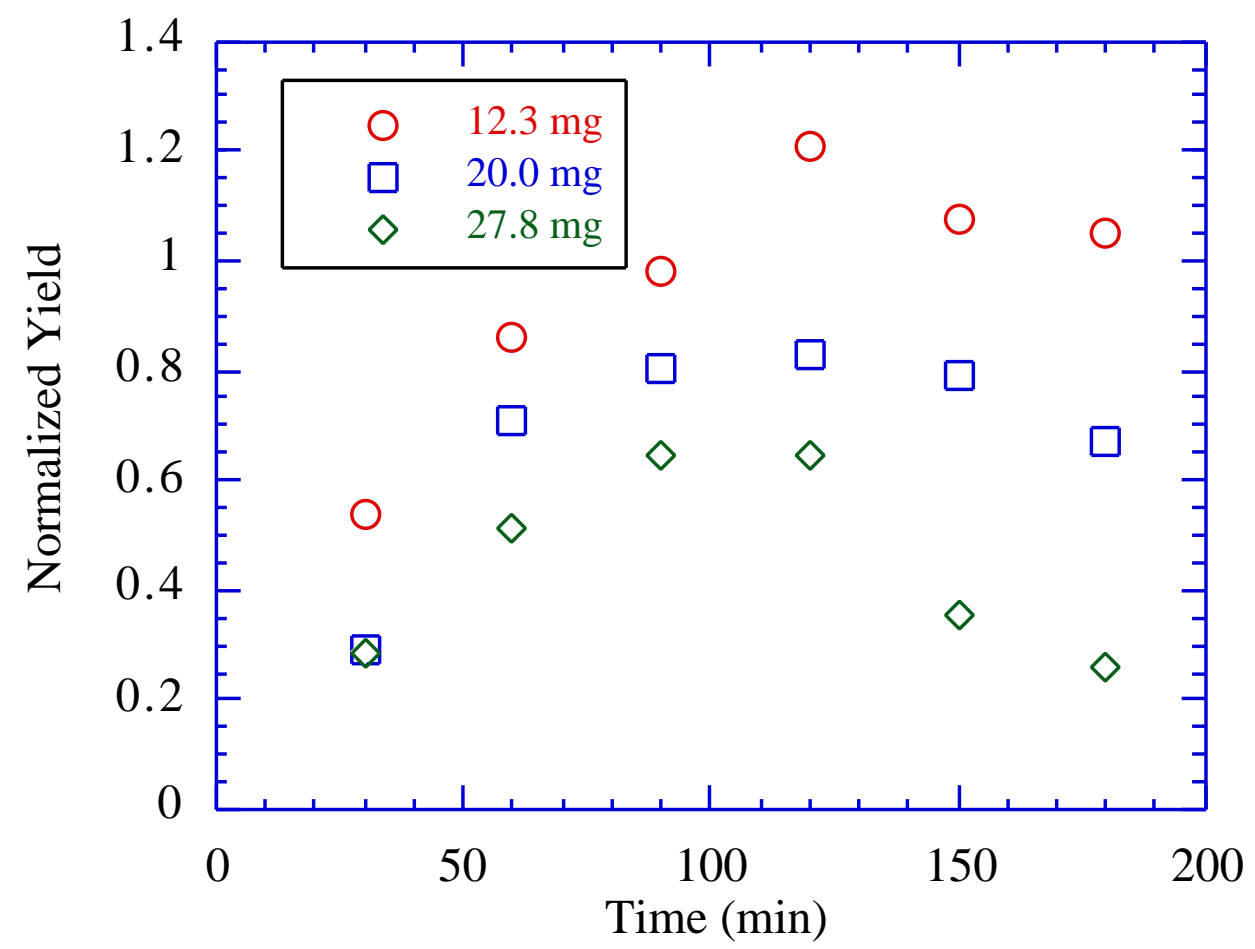

Figure 20: Comparison of normalized yields of tetracosene as a function of reactant loading for reactions with HDPE.

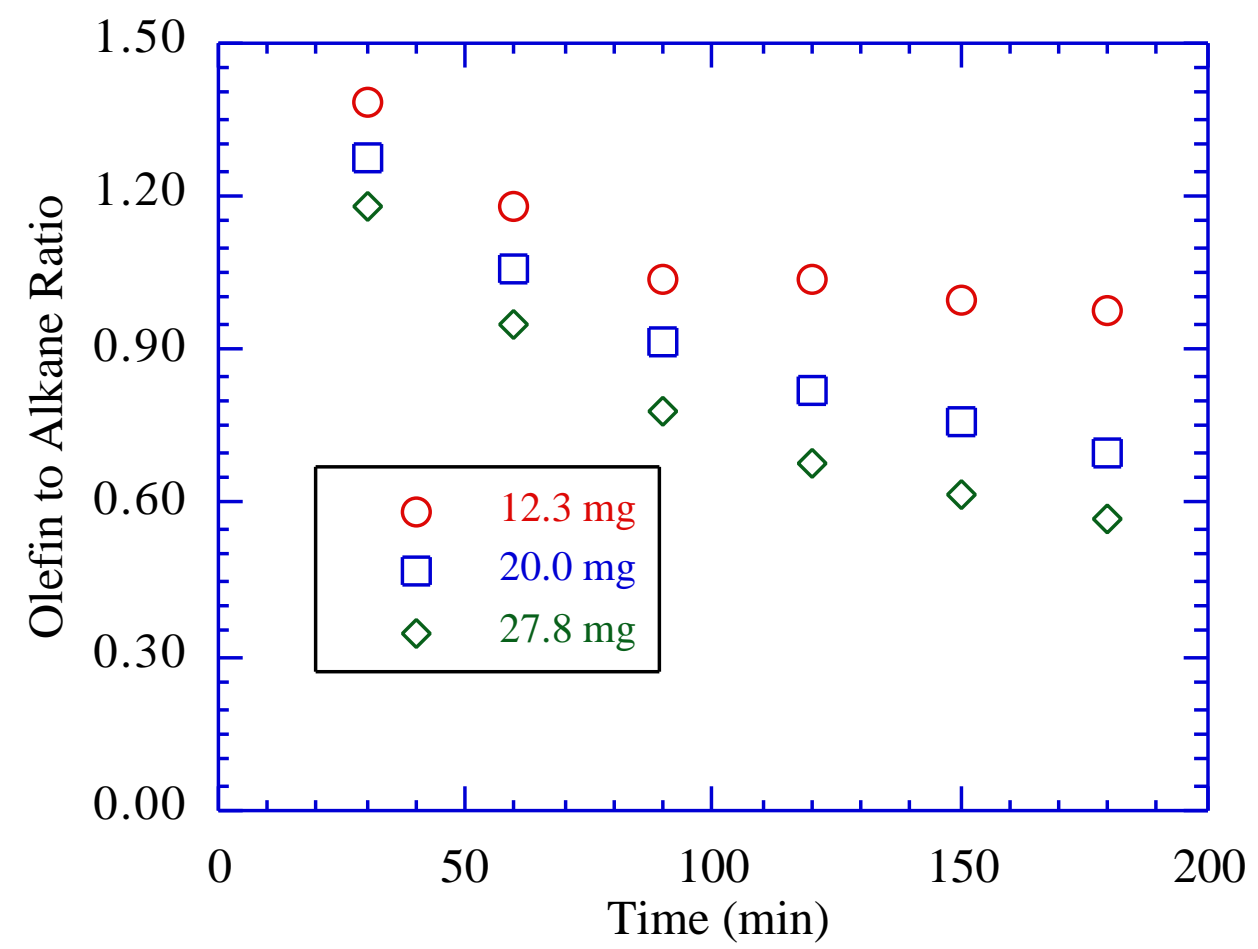

Figure 21: Comparison of $\alpha$-olefin to n-alkane ratio for $\mathrm{C}_{18}$ as a function of reactant loading for reactions with HDPE. 
tetradecane. For instance, the olefin to paraffin ratio changed 0.3 as compared to 0.1 for $\mathrm{C}_{11}$ products during the $27.8 \mathrm{mg}$ HDPE and C14 experiments, respectively. The differences in product ratios for HDPE and tetradecane pyrolyses can partially be attributed to effects associated with the initial molecular weight of the reactants. With longer hydrocarbons, there may be more pathways for favorable bimolecular reactions, due to both the increase in the number of secondary carbons and the fact that the pyrolysis is occurring in both the gaseous and condensed phases. These factors will result in higher proportions of paraffins and internal olefins versus $\alpha$-olefins. However, direct comparison is not possible without a common measure of conversion of the two reactants.

Overall, pyrolysis of HDPE resulted in a slightly broader product distribution and wider range of product yields than those observed for tetradecane pyrolysis. In general, however, the primary reaction mechanisms and products are similar for tetradecane and HDPE pyrolysis, indicating that tetradecane is an appropriate model for HDPE.

\section{Conclusions}

Recent investigations have demonstrated the feasibility of coprocessing of coal with polymers. In this study, feedstock interactions were observed using model compound mimics of both coal and polyethylene. In binary mixtures, the conversion of tetradecane increased while the selectivity to primary products of NBBM pyrolysis was enhanced. These observations were attributed to the stabilization of NBBM-derived radicals through hydrogen abstraction from tetradecane which in turn, increased the rate of tetradecane conversion.

The initial loading of each reactant and overall loading were found to affect both the conversion of tetradecane and the overall product distribution during coprocessing. As the NBBM to tetradecane ratio was raised, only a slight difference in reactant conversions was observed, but a significant change in the selectivity towards primary products from NBBM occurred. The higher proportion of NBBM resulted in an increase of self-interactions, which resulted in a larger quantity of retrograde condensation products. Increasing the initial charge of both reactants resulted in a significant enhancement of tetradecane reactivity. The primary product yields from the coal model compound showed a slight decrease when compared to the reactions conducted with the same reactant ratio but lower total loading. However, the values were higher than those observed for the reactions carried out with a larger ratio of NBBM to tetradecane. These effects on conversions and selectivities were attributed to an increase in bimolecular reactions at higher concentrations.

In order to optimize the interaction between reactants, further experimental and theoretical studies will be conducted at high pressures (already in progress) and in the presence of hydrogen donor solvents and catalysts in order to delineate the underlying kinetics, pathways, and mechanism controlling coal/polymer coprocessing.

\section{Path Forward}

Work carried out in the next six months will focus on high-pressure pyrolysis of the coal and polymer model compounds in both nitrogen and hydrogen. These experiments will provide insight into the reaction pathways and mechanisms which dominate the interactions of these reactants at high pressures. Once this data has been obtained, the effects of adding hydrogen donor solvents and catalysts to the system may be addressed. In addition, reactions with real feedstocks will be continued in parallel in order to obtain complimentary information which may be used as a measure of both extrinsic effects and the applicability of the model compound studies. 


\section{Acknowledgments}

The authors would like to thank Naresh Shaw and Gerald Huffman from the University of Kentucky and Dady Dadyburjor from West Virginia University for their insightful comments and help in designing a high pressure delivery system. Undergraduates Mike Dennis and Daniel Prekup also deserve credit for conducting some of the low pressure pyrolysis experiments.

\section{Manuscripts and Presentations}

De Witt, M.J. and Broadbelt, L.J., "Coprocessing of Polymeric Waste with Coal: Reaction of Polyethylene and Coal Model Compounds", Preprints of the American Chemical Society, Division of Fuel Chemistry, San Francisco, CA, April 1997.

Broadbelt, L.J., "Polymer Resource Recovery Through Coprocessing: Thermal and Catalytic Chemistry", Invited Lecture, Department of Chemical Engineering, The Ohio State University, Columbus, OH, May 1997.

Broadbelt, L.J., "Polymer Resource Recovery Through Coprocessing", Invited Lecture, Department of Civil Engineering, Northwestern University, Evanston, IL, April 1997.

De Witt, M.J. and Broadbelt, L.J., "Coprocessing of Polymeric Waste with Coal: Reaction of Polyethylene and Coal Model Compounds", Annual Meeting of the American Chemical Society, Division of Fuel Chemistry, San Francisco, CA, April 1997.

Broadbelt, L.J., Woo, O.S. and De Witt, M.J., "Polymer Resource Recovery Through Coprocessing: Thermal and Catalytic Chemistry", Invited Lecture, Catalysis Club of Chicago, Chicago, IL, April 1997.

\section{References}

(1) Rowatt, R. J., Chemtech, 1993, 23(1), 56-60.

(2) Taghei, M., Feng, Z., Huggins, F., and Huffman, G. R., Energy \& Fuels, 1994, 8, 1228-1232.

(3) Menges, G., Emminger, H., and Lackner, G., International Journal of Materials and Product Technology, 1991, 6(4), 307-330.

(4) Graff, G., Modern Plastics, 1992, 69(7), 45.

(5) Fouhy, K., Kim, I., Moore, S., and Culp, E., Chemical Engineering, 1993, 100(12), 30-33.

(6) EPA, U.S. Environmental Protection Agency, 1992.

(7) Joo, H. K., Curtis, C.W., Energy \& Fuels, 1997, 11(4), 801-812.

(8) Rothenberger, K. S., Cugini, A.V., Thompson, R.L., Ciocco, M.V., Energy \& Fuels, 1997, 11(4), 849-855.

(9) Farcasiu, M. and Smith, C., Energy \& Fuels, 1991, 5(1), 83-87.

(10) Matson, D. W., Linehan, J. C., Darab, J. G., and Buehler, M. F., Energy \& Fuels, 1994, 8, 10-18.

(11) Tang, Y. and Curtis, C. W., Energy \& Fuels, 1994, 8, 63.

(12) Walter, T. D., Casey, S. M., Klein, M. T., and Foley, H. C., Catalysis Today, 1994, 19, 367-380.

(13) Rice, F. O., Journal of the American Chemical Society, 1933, 55, 3035-3040.

(14) Kossiakoff, A. and Rice, F. O., Journal of American Chemical Society, 1943, 65, 590-595.

(15) Voge, H. H. and Good, G. M., Journal of American Chemical Society, 1949, 71, 593-597. 
(16) Mushrush, G. W. and Hazlett, R. N., Industrial \& Engineering Chemistry Fundamentals, 1984, 23, 288-294.

(17) LaMarca, C., Libanati, C., and Klein, M. T., Chemical Engineering Science, 1990, 45(8), 2059-2065.

(18) Poutsma, M. L., Energy \& Fuels, 1990, 4(2), 113-131.

(19) Fabuss, B. M., Smith, J. O., and Satterfield, C. N., Industrial Engineering Process Design and Development, 1962, 1, 293-299.

(20) Song, C., Lai, W. C., and Schobert, H. H., Industrial \& Engineering Chemistry Research, 1994, 33, 534-547.

(21) Albright, L. F., Crynes, B. L., and Corcoran, W. H., in 'Pyrolysis: Theory and Industrial Practice', New York, 1983.

(22) Ng, S. W., Seoud, H., Stanciulescu, M., Sugimoto, Y, Energy \& Fuels, 1995, 9, 735-742. 